\title{
Analyse de la dégradation thermique du Poly(éther éther cétone)
}

\author{
Emilie Courvoisier ${ }^{1,2}$, Yoann Bicaba ${ }^{1}$, and Xavier Colin ${ }^{2, *}$ \\ 1 SAFRAN Composites, 33 avenue de la Gare, 97601 Itteville, France \\ 2 Laboratoire PIMM, Arts et Métiers ParisTech, 151 boulevard de l'Hôpital, 75013 Paris, France
}

Reçu le 29 août 2017 / Accepté le 19 janvier 2018

\begin{abstract}
Résumé. La dégradation thermique du PEEK a été étudiée à l'état caoutchoutique dans de larges intervalles de température (entre 180 et $320^{\circ} \mathrm{C}$ ) et de pression partielle d'oxygène (entre 0,21 et 50 bars). Tout d'abord, les mécanismes de vieillissement thermique ont été analysés et élucidés par spectroscopie IRTF et par calorimétrie différentielle (DSC) sur des films de PEEK suffisamment minces (entre 10 et $60 \mu \mathrm{m}$ d'épaisseur) pour s'affranchir totalement des effets de la diffusion d'oxygène. L'oxydation se produit sur les cycles aromatiques provoquant la croissance de cinq nouvelles bandes d'absorption IR centrées à $3650,3525,1780,1740$ et $1718 \mathrm{~cm}^{-1}$ et attribuées aux vibrations d'élongation des liaisons $\mathrm{O}-\mathrm{H}$ du phénol et de l'acide benzoïque, et des liaisons $\mathrm{C}-\mathrm{O}$ de l'anhydride benzoïque, du benzoate de phényle et de la fluorénone respectivement. De plus, l'oxydation conduit à une large prédominance de la réticulation sur les coupures de chaîne (augmentation de $T_{\mathrm{g}}$ ) empêchant le recuit du PEEK, en particulier lorsque la température d'exposition est supérieure au pied du pic de fusion. Enfin, les conséquences de l'oxydation sur les propriétés élastiques ont été analysées et élucidées par micro-indentation sur des sections droites préalablement polies de plaquettes de PEEK de $3 \mathrm{~mm}$ d'épaisseur. Les variations du module d'Young et du taux de cristallinité se corrèlent parfaitement, vérifiant ainsi la relation de Tobolsky.
\end{abstract}

Mots clés : PEEK / thermo-oxydation / coupures de chaîne / réticulation / module d'Young

\begin{abstract}
Analytical study of the thermal degradation of poly(ether ether ketone). The thermal degradation of PEEK has been studied in rubbery state in wide ranges of temperature (between 180 and $320^{\circ} \mathrm{C}$ ) and oxygen partial pressure (between 0.21 and 50 bars). First of all, the thermal ageing mechanisms have been analysed and elucidated by FTIR spectroscopy and by differential scanning calorimetry (DSC) on sufficiently thin PEEK films (between 10 and $60 \mu \mathrm{m}$ thickness) to be totally free of the effects of oxygen diffusion. Oxidation occurs on aromatic rings causing the growth of five new IR absorption bands centered at 3650, 3525, 1780, 1740 and $1718 \mathrm{~cm}^{-1}$ and attributed to the stretching vibrations of the $\mathrm{O}-\mathrm{H}$ bonds of phenol and benzoïc acid, and the $\mathrm{C}-\mathrm{O}$ bonds of benzoïc anhydride, phenyl benzoate and fluorenone respectively. In addition, oxidation leads to a large predominance of crosslinking over chain scissions (increase in $\mathrm{T}_{\mathrm{g}}$ ) preventing the annealing of PEEK, in particular when the temperature of exposure is higher than the onset of the melting endotherm. Finally, the consequences of oxidation on the elastic properties have been analysed and elucidated by micro-indentation on preliminarily polished cross-sections of PEEK plates of $3 \mathrm{~mm}$ thickness. The changes in Young's modulus and crystallinity ratio correlate perfectly, thus checking the Tobolsky's relationship.
\end{abstract}

Keywords: PEEK / thermal oxidation / chain scissions / crosslinking / Young's modulus

\section{Introduction}

La compétition sur le marché aéronautique dépend d'enjeux à la fois économiques et écologiques tels que la réduction des coûts, de la masse embarquée et de la consommation de carburant. C'est pourquoi, depuis son origine, l'industrie aéronautique est à la recherche de nouveaux matériaux plus légers aux propriétés thermo-

\footnotetext{
* e-mail: xavier.colin@ensam.eu
}

mécaniques élevées. Dans ce contexte, l'emploi de matériaux composites constitués de matrice organique renforcée par des fibres de verre ou de carbone (CMO) n'a cessé de progresser dans le but de remplacer peu à peu les matériaux métalliques. D'abord conçus pour la fabrication de pièces de structure (ailes, queue, fuselage, etc.), les CMO sont maintenant envisagés pour des applications dans des environnements thermochimiques de plus en plus sévères, comme les zones proches des sources de chaleur (environnements de moteur d'avion). Cependant, ils ne pourront être utilisés que si leur durabilité à long terme est 
clairement démontrée. L'utilisation de matrices thermoplastiques présente plusieurs avantages, comme l'intégration de fonction ou encore l'assemblage de pièces de même nature ou de natures différentes. Parmi les matrices thermoplastiques pouvant répondre au cahier des charges, le poly(éther éther cétone) (PEEK) apparaît comme un sérieux candidat. Il s'agit d'un polymère semi-cristallin de structure aromatique lui confèrant à la fois de hautes performances thermomécaniques $\left(\mathrm{T}_{\mathrm{g}} \approx 153^{\circ} \mathrm{C}\right.$ et $\mathrm{T}_{\mathrm{f}} \approx$ $343^{\circ} \mathrm{C}$ ) et une grande résistance chimique à la plupart des solvants usuels (acétone, toluène, acétate d'éthyle, etc.) et aux fluides aéronautiques (en particulier, le Skydrol). La dégradation thermique du PEEK a été étudiée sous atmosphère inerte ou faiblement oxygénée (typiquement dans l'air) surtout à l'état fondu (entre 340 et $485^{\circ} \mathrm{C}$ ), mais rarement à l'état caoutchoutique (entre 300 et $320^{\circ} \mathrm{C}$ ) et jamais, à notre connaissance, à l'état vitreux.

L'oxydation du PEEK se produit sur les seuls groupes hydrocarbonés de l'unité monomère, c'est-à-dire sur les cycles aromatiques, malgré la très faible labilité des atomes d'H. En effet, l'énergie de dissociation de la liaison $\mathrm{C}-\mathrm{H}$ aromatique est de l'ordre de $465 \mathrm{~kJ} . \mathrm{mol}^{-1}$, contre 393 et $378 \mathrm{~kJ} \cdot \mathrm{mol}^{-1}$ pour les liaisons $\mathrm{C}-\mathrm{H}$ méthylénique et méthynique respectivement [1]. Au-delà de $400^{\circ} \mathrm{C}$, l'oxydation serait principalement amorcée par la rupture des liaisons éther et cétone de l'unité monomère [2-4]. Elle conduit à la disparition des groupes cétone (à $1653 \mathrm{~cm}^{-1}$ ), mais aussi à la formation d'une grande variété de produits de dégradation détectés par spectroscopie IRTF. Il s'agit notamment de produits macromoléculaires comme les phénols (entre 3400 et $3700 \mathrm{~cm}^{-1}$ ), le benzoate de phényle (à $\left.1739 \mathrm{~cm}^{-1}\right)$ et la fluorénone $\left(1711 \mathrm{~cm}^{-1}\right)$ [2-4], mais aussi de nombreux composés volatils [4-6]. Difficilement détectables en dessous de $430{ }^{\circ} \mathrm{C}$, ces derniers ont été mis clairement en évidence au-dessus de $450{ }^{\circ} \mathrm{C}$ par analyse gravimétrique couplée à la chromatographie en phase gazeuse (ATG-CPG) ou à la spectrométrie de masse (ATG-SM). Il s'agit de quinones, de phénols, du monoxyde et du dioxyde de carbone, du benzène, mais aussi d'éthers et de cétones aromatiques [4,6].

Des mécanismes impliquant le réarrangement des radicaux phénoxyle et acyle, issus de la rupture des liaisons éther et cétone respectivement, ont été proposés pour expliquer la formation de la plupart des produits de dégradation. Cependant, ces mécanismes purement thermolytiques ne permettent pas d'expliquer l'accélération de la cinétique d'oxydation avec l'élévation de la pression partielle d'oxygène. Entre 360 et $460^{\circ} \mathrm{C}$, une seconde importante source de radicaux, pouvant elle aussi expliquer l'accumulation de plusieurs produits de dégradation, serait la décomposition unimoléculaire des hydroperoxydes [7].

Des modifications de l'architecture macromoléculaire du PEEK ont aussi été détectées par chromatographie d'exclusion stérique (CES ou CPG) [5], et par viscosimétrie en solution [3] ou à l'état fondu $[5,7,8]$. Entre 300 et $485^{\circ} \mathrm{C}$, la réticulation prédomine largement sur les coupures de chaîne, cette prédominance relative étant amplifiée à plus forte pression partielle d'oxygène. La réticulation se traduit par une augmentation de la masse moléculaire $[3,5,7,8]$, de la fraction de gel $[3,4]$ et de la température de transition vitreuse $[5,8,9]$. Le mécanisme de réticulation le plus probable est la recombinaison bimoléculaire des radicaux phényle [3-6].

Enfin, des modifications de la morphologie cristalline du PEEK ont été mises en évidence par analyse calorimétrique (DSC). Entre 380 et $440^{\circ} \mathrm{C}$, la réticulation limite la cristallisation quand le PEEK est refroidi à température ambiante depuis son état fondu, ce qui se traduit par une diminution de son point de fusion et de son taux de cristallinité $[5,8,9]$.

Cet article présente une analyse à la fois multi-échelles et multi-techniques de la dégradation thermique du PEEK à des températures plus basses et des pressions partielles d'oxygène plus fortes que celles étudiées jusqu'à présent dans la littérature. Une attention particulière est portée aux conséquences de la dégradation sur deux propriétés thermomécaniques clés en aéronautique : la température de transition vitreuse et le module d'Young.

\section{Matériaux et techniques}

Le matériau d'étude est le PEEK 150G fourni par la société Victrex sous forme de granulés. Des plaques de $3 \mathrm{~mm}$ d'épaisseur ont été injectées au Pôle Européen de Plasturgie (PEP) d'Oyonnax à partir de ces granulés. Ces plaques ont été ensuite découpées au microtome LEICA RM2255 pour obtenir des films suffisamment minces (typiquement entre 10 et $60 \mu \mathrm{m}$ d'épaisseur) garantissant une oxydation homogène dans toute l'épaisseur. La cinétique d'oxydation du PEEK a été étudiée à l'état caoutchoutique à 180, 200, 220, 250, 280, 300 et $320^{\circ} \mathrm{C}$ sous des pressions partielles d'oxygène comprises entre 0,21 bar (dans des étuves à ventilation d'air forcée, régulées à $\pm 1^{\circ} \mathrm{C}$ ) et 50 bars (dans des autoclaves). Compte tenu du fort impact de la pression partielle d'oxygène sur la cinétique d'oxydation, les vieillissements aux températures les plus hautes (typiquement entre 280 et $320^{\circ} \mathrm{C}$ ) ont été menés sous 0,21 bar d'oxygène, alors que ceux aux températures les plus basses (entre 180 et $250^{\circ} \mathrm{C}$ ) ont dû être réalisés au-dessus de 1 bar d'oxygène pour pouvoir détecter des changements structuraux significatifs sur des durées d'étude compatibles avec la durée d'un travail de thèse (de quelques années). Tous les films ont été périodiquement sortis des enceintes de vieillissement et refroidis à température ambiante dans un dessiccateur contenant du silica-gel pour éviter une reprise en humidité avant d'être caractérisés. À noter que des échantillons plus épais (plaquettes de 3 mm d'épaisseur) ont aussi été exposés à 280 et $300^{\circ} \mathrm{C}$ dans des étuves ventilées pour mettre en évidence des profils d'oxydation. Dans tous les cas, les conséquences de l'oxydation ont été analysées par plusieurs techniques complémentaires depuis l'échelle moléculaire jusqu'à l'échelle macroscopique, en passant par les échelles macromoléculaire, morphologique et microscopique.

Les modifications de la structure moléculaire ont été suivies par spectroscopie IRTF en mode transmission. Les spectres IRTF des films de PEEK ont été mesurés avant et après vieillissement afin de pouvoir détecter et quantifier non seulement la disparition des groupes chimiques initialement présents dans la matrice PEEK, mais aussi la formation des principaux produits d'oxydation. Les analyses ont été réalisées à l'aide d'un appareil Frontier de 

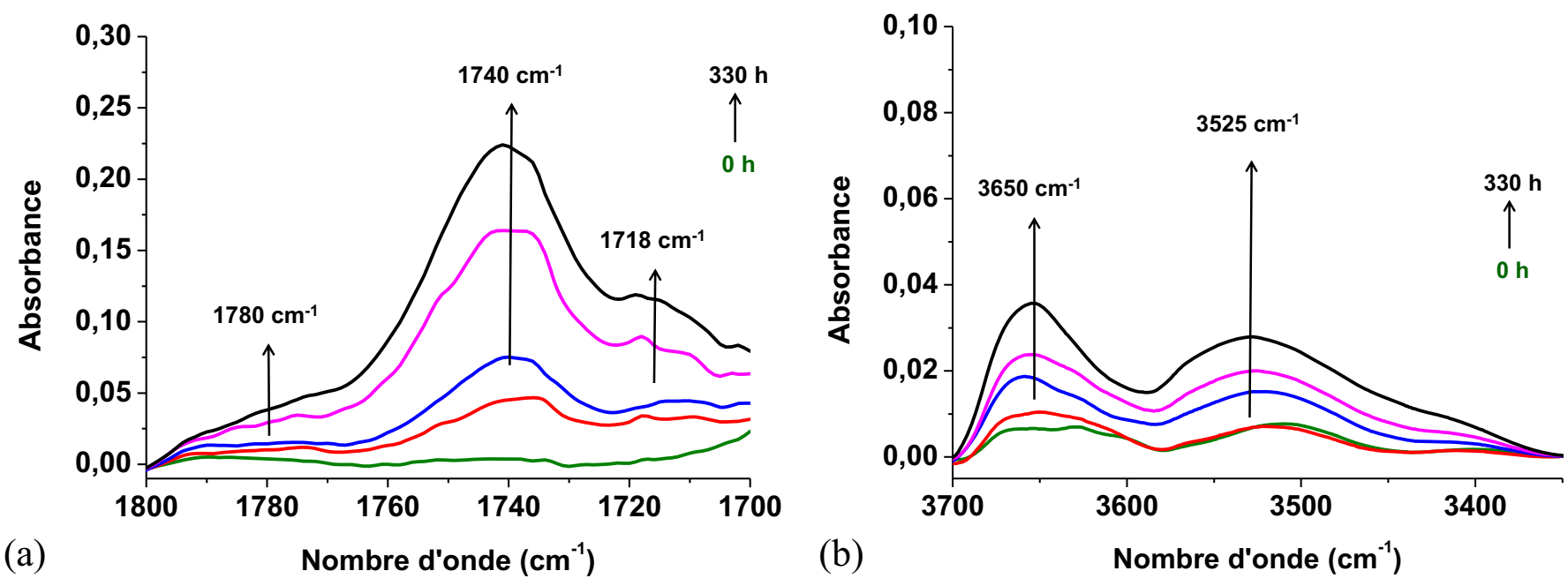

Fig. 1. Modification des régions des liaisons $\mathrm{C}=\mathrm{O}(\mathrm{a})$ et $\mathrm{O}-\mathrm{H}(\mathrm{b})$ du spectre IRTF du PEEK au cours de son vieillissement thermique dans l'air à $300^{\circ} \mathrm{C}$.

Fig 1. Changes in the regions of $C=O(a)$ and $O-H$ bonds (b) in the FTIR spectrum of PEEK during its thermal ageing in air at $300^{\circ} C$.

chez Perkin Elmer dans un domaine spectral s'étendant de 400 à $4000 \mathrm{~cm}^{-1}$, après avoir moyenné les 16 enregistrements obtenus avec une résolution minimale de $4 \mathrm{~cm}^{-1}$. Le vieillissement thermique du PEEK conduit à deux principales modifications du spectre IRTF (Fig. 1). Tout d'abord, dans la zone des hydroxyles entre 3400 et $3700 \mathrm{~cm}^{-1}$, on note l'apparition et la croissance de deux bandes d'absorption IR centrées à 3600 et $3525 \mathrm{~cm}^{-1}$. Elles sont respectivement attribuées aux vibrations d'élongation des liaisons $\mathrm{O}-\mathrm{H}$ des phénols et de tous les autres types de produits hydroxyles, majoritairement l'acide benzoïque $[2,10]$. Ensuite, dans la zone des carbonyles entre 1700 et $1800 \mathrm{~cm}^{-1}$, on observe l'apparition et la croissance de trois bandes d'absorption IR centrées à 1780 , 1740 et $1718 \mathrm{~cm}^{-1}$. Elles sont respectivement attribuées aux vibrations d'élongation des liaisons $\mathrm{C}-\mathrm{O}$ de l'anhydride benzoïque, du benzoate de phényle et de la fluorénone $[2,10,11]$.

Rappelons que la concentration $\mathrm{C}$ des différents produits de dégradation est liée à l'absorbance Abs de leur bande IR par la loi de Beer-Lambert:

$$
C=\frac{A b s}{e \times \varepsilon},
$$

où e est l'épaisseur de l'échantillon $(\mathrm{cm})$ et $\varepsilon$ le coefficient d'extinction molaire dont les valeurs moyennes sont accessibles dans la littérature: $\varepsilon(\mathrm{O}-\mathrm{H}$ des phénols et acides $)=90 \mathrm{~L} \cdot \mathrm{mol}^{-1} \cdot \mathrm{cm}^{-1}[12,13], \varepsilon(\mathrm{C}=\mathrm{O}$ des anhydrides et cétones $)=300 \mathrm{~L} \cdot \mathrm{mol}^{-1} \cdot \mathrm{cm}^{-1}[14]$ et $\varepsilon(\mathrm{C}=\mathrm{O}$ des esters $)=$ $550 \mathrm{~L} \cdot \mathrm{mol}^{-1} \cdot \mathrm{cm}^{-1}[15]$.

Signalons que, en raison du chevauchement des bandes d'absorption IR dans la région des carbonyles, il a été nécessaire de réaliser une déconvolution mathématique basée sur des fonctions gaussiennes à l'aide du logiciel OriginLab pour pouvoir extraire les contributions élémentaires des différents groupes carbonyle (Fig. 2). En plus des trois bandes précedentes, cette déconvolution a mis en évidence une quatrième contribution vers $1790 \mathrm{~cm}^{-1}$.
Cependant, comme son absorbance est extrêment faible et n'évolue pas pendant le vieillissement thermique, cette dernière bande a été logiquement négligée par la suite.

Les conséquences de l'oxydation sur l'architecture macromoléculaire et la morphologie cristalline ont été suivies par analyse calorimétrique (DSC). Les thermogrammes DSC des films de PEEK ont été mesurés avant et après vieillissement afin d'accéder aux évolutions de la température de transition vitreuse $\left(\mathrm{T}_{\mathrm{g}}\right)$, du point de fusion $\left(\mathrm{T}_{\mathrm{f}}\right)$ et de l'enthalpie de fusion $\left(\Delta \mathrm{H}_{\mathrm{f}}\right)$. Les analyses ont été effectuées à l'aide d'un calorimètre Q1000 de chez TA Instruments entre 25 et $300^{\circ} \mathrm{C}$ avec une vitesse de chauffe de $10^{\circ} \mathrm{C} \cdot \mathrm{min}^{-1}$ sous flux d'azote. Les valeurs de $\mathrm{T}_{\mathrm{g}}$ et $\mathrm{T}_{\mathrm{f}}$ ont été prises au point d'inflexion de la courbe de variation du flux de chaleur avec la température et au sommet du pic endothermique respectivement. La valeur de $\Delta \mathrm{H}_{\mathrm{f}}$ correspond à l'aire sous le pic endothermique.

Le taux de cristallinité a été déterminé à l'aide de la relation suivante:

$$
X_{C}(\%)=\frac{\Delta H_{f}}{\Delta H_{f 0}} \times 100,
$$

où $\Delta \mathrm{H}_{\mathrm{f} 0}$ est l'enthalpie de fusion du cristal : $\Delta \mathrm{H}_{\mathrm{f} 0}=130{\mathrm{~J} . \mathrm{g}^{-1}}^{-1}$ pour le PEEK [16].L'épaisseur des lamelles cristallines a été déterminée à l'aide de la relation de Gibbs-Thomson :

$$
l_{c}=\frac{2 \sigma_{e} T_{f 0}}{\rho_{c} \Delta H_{f 0}\left(T_{f 0}-T_{f}\right)},
$$

où $\sigma_{\mathrm{e}}$ est l'énergie libre de surface à l'interface phase amorphe/phase cristalline, $\rho_{\mathrm{C}}$ est la densité de la phase cristalline et $\mathrm{T}_{\mathrm{fo}}$ est le point de fusion du cristal parfait : $\sigma_{\mathrm{e}}=4,9 \times 10^{-2} \mathrm{~J} \cdot \mathrm{m}^{-2}, \rho_{\mathrm{C}}=1400 \mathrm{~kg} \cdot \mathrm{m}^{-3}$ et $\mathrm{T}_{\mathrm{f} 0}=668 \mathrm{~K}$ pour le PEEK [16].

Enfin, l'impact du vieillissement sur les propriétés élastiques (principalement sur le module d'Young) a été déterminé par des essais de micro-indentation sur les 

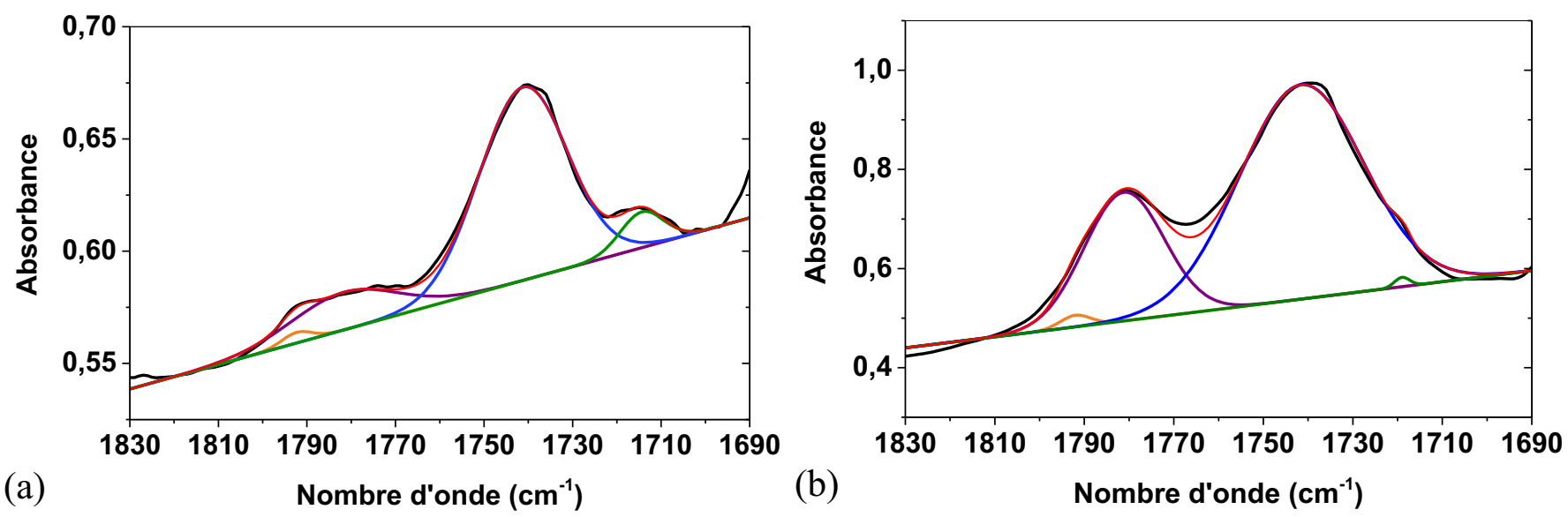

Fig. 2. Déconvolution de la région des liaisons $\mathrm{C}=\mathrm{O}$ du spectre $\mathrm{IR}$ du $\mathrm{PEEK}$ après $324 \mathrm{~h}$ d'exposition à $300^{\circ} \mathrm{C}$ sous 0,21 bar d'oxygène (a) et après $196 \mathrm{~h}$ d'exposition à $250^{\circ} \mathrm{C}$ sous 10 bars d'oxygène $(\mathrm{b})$.

Fig 2. Deconvolution of the region of $C=O$ bonds in the IR spectrum of PEEK after 324 h of exposure at $300^{\circ} \mathrm{C}$ under 0.21 bar of oxygen (a) and after $196 \mathrm{~h}$ of exposure at $250^{\circ} \mathrm{C}$ under 10 bars of oxygen (b).

sections droites préalablement polies des plaquettes de $3 \mathrm{~mm}$ d'épaisseur. Les plaquettes ont été découpées suivant leur épaisseur et enrobées à froid dans une résine acrylique commerciale $\mathrm{KM}-\mathrm{V}$, qui a ensuite été réticulée pendant $12 \mathrm{~h}$ sous vide primaire à température ambiante. Les sections droites ont été polies à l'aide d'une polisseuse MECAPOL P320 en utilisant tout d'abord, une série de papiers abrasifs en particules de carbure de silicium et de taille décroissante (typiquement de granulométrie 80 à 2400). Enfin, une finition miroir a été obtenue à l'aide de pâtes de particules de diamant de taille décroissante (typiquement de 3 à $0,25 \mu \mathrm{m}$ ). Les indentations ont ensuite été réalisées à l'aide du Micro Indentation Tester (MHT) de chez CSM Instrument équipé d'une pointe en diamant de type Vickers de géométrie pyramidale avec une force de $500 \mathrm{mN}$, et des vitesses de charge et décharge de $1000 \mu \mathrm{m} \cdot \mathrm{min}^{-1}$. Une pause de 5 secondes a été systématiquement appliquée entre la charge et la décharge. Le logiciel d'exploitation Indentation 4.37 donne directement accès à la valeur du module réduit du matériau $\left(\mathrm{E}_{\mathrm{r}}\right)$ qui est calculée selon la méthode d'Oliver and Pharr [17-19]:

$$
E_{r}=\frac{\sqrt{\pi} S}{2 \beta \sqrt{A_{c}}},
$$

où $\mathrm{S}$ est la pente initiale de la courbe de décharge, $\beta$ est un facteur de forme dépendant du type d'indenteur $(\beta=1,012$ dans le cas d'un indenteur de type Vickers) et $\mathrm{A}_{\mathrm{c}}$ est l'aire de contact projetée. Cette dernière grandeur est directement donnée par le logiciel d'exploitation. Elle dépend de la profondeur de contact de l'indenteur sur l'échantillon et de la géométrie de l'indenteur.

Le module élastique local a été déterminé à partir du module réduit à l'aide de la relation suivante:

$$
E=\frac{1}{\frac{1-v^{2}}{E_{r}}-\frac{1-v_{i}^{2}}{E_{i}}},
$$

où $v$ est le coefficient de Poisson du PEEK vierge égal à 0,4 (donnée fournisseur), et $\nu_{\mathrm{i}}$ et $\mathrm{E}_{\mathrm{i}}$ sont le coefficient de Poisson et le module d'Young de l'indenteur en diamant, respectivement égaux à 0,07 et $1141 \mathrm{GPa}$.

Le module élastique global $\mathrm{E}_{\text {Echant }}$ de l'échantillon a ensuite été déduit des $\mathrm{N}$ valeurs locales $\mathrm{E}(\mathrm{j})$ composant le profil de micro-indentation à l'aide de la relation suivante:

$$
\mathrm{E}_{\mathrm{Echant}}=\frac{1}{N} \sum_{j=1}^{N} E(j) .
$$

Dans cette étude, $\mathrm{E}_{\text {Echant }}$ a été assimilé au module d'Young de l'échantillon après avoir vérifié, par traction uniaxiale, qu'il donne une très bonne estimation de ce dernier. En effet, il a été trouvé que leurs valeurs respectives ne diffèrent que d'un facteur 0,84 .

\section{Résultats et discussion}

\subsection{Modifications de la structure moléculaire}

À titre d'exemple, les figures 3 et 4 rapportent l'évolution des concentrations des différents produits carbonyle: l'anhydride benzoïque (à $1780 \mathrm{~cm}^{-1}$ ), le benzoate de phényle $\left(1740 \mathrm{~cm}^{-1}\right)$ et la fluorénone $\left(1718 \mathrm{~cm}^{-1}\right)$, ainsi que des différents produits hydroxyle: les phénols $\left(3650 \mathrm{~cm}^{-1}\right)$ et l'acide benzoïque $\left(3525 \mathrm{~cm}^{-1}\right)$, au cours du vieillissement thermique du PEEK dans l'air entre 250 et $320^{\circ} \mathrm{C}$. Comme attendu, la température accélère la cinétique d'oxydation du PEEK.

Deux indicateurs de vieillissement ont été déterminés graphiquement pour l'ensemble des courbes cinétiques. Il s'agit du temps d'induction $\left(t_{i}\right)$ marquant le début de la phase d'auto-accélération, et de la vitesse maximale d'oxydation $\left(\mathrm{r}_{\mathrm{ox}}\right)$ correspondant à la pente maximale des courbes cinétiques. Ces deux indicateurs obéissent à une loi d'Arrhenius entre 250 et $320^{\circ} \mathrm{C}$. Malgré la dispersion des résultats, les énergies d'activation de $t_{i}$ et $r_{o x}$ sont du même ordre de grandeur pour l'ensemble des produits de 

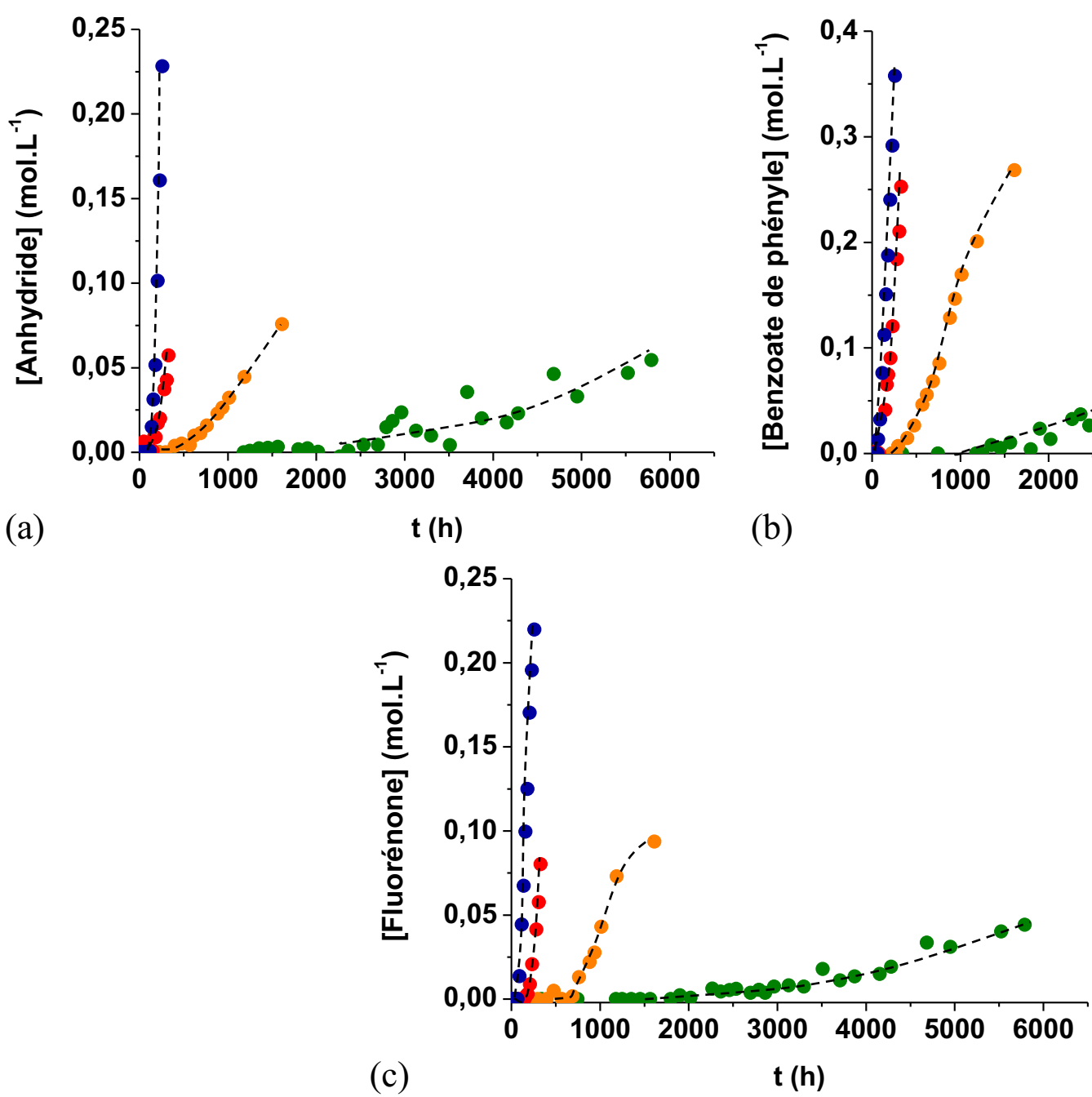

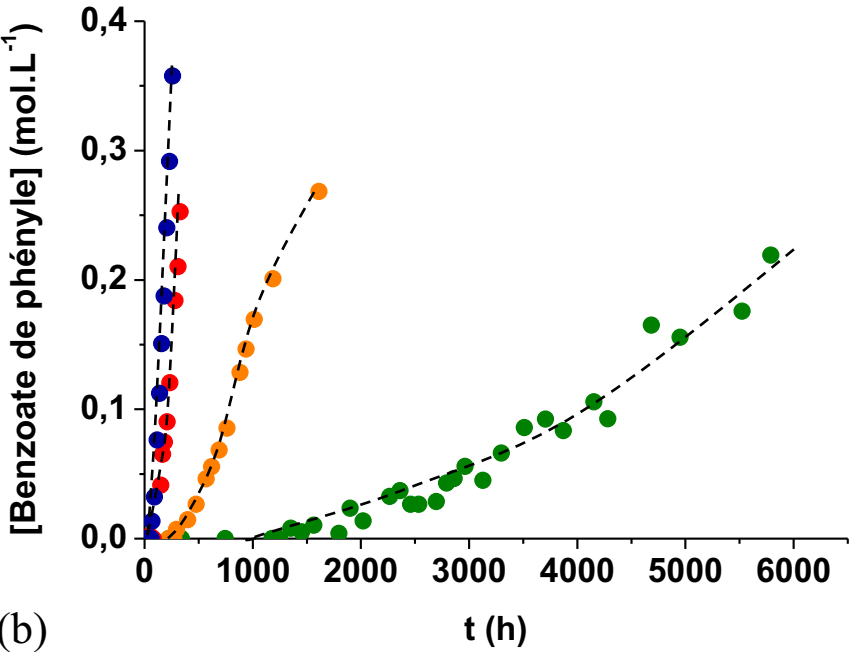

t (h)

Fig. 3. Évolution des concentrations en anhydride benzoïque (a), benzoate de phényle (b) et fluorénone (c) au cours du vieillissement thermique du PEEK dans l'air à 250 (en vert), 280 (orange), 300 (rouge) et $320^{\circ} \mathrm{C}$ (bleu).

Fig 3. Changes in the concentrations of benzoic anhydride (a), phenyl benzoate (b) and fluorenone (c) during the thermal ageing of PEEK in air at 250 (in green), 280 (orange), 300 (red) and $320^{\circ} \mathrm{C}$ (blue).

dégradation. À titre d'exemple, le tableau 1 récapitule les valeurs d'énergie d'activation trouvées dans l'air. Les valeurs moyennes, qui caractérisent la cinétique d'oxydation du PEEK dans ces conditions d'exposition, sont:

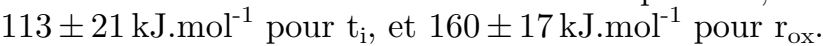

Les vitesses maximales d'accumulation du benzoate de phényle et de la fluorénone dans l'air à l'état fondu, typiquement entre 400 et $485^{\circ} \mathrm{C}$, ont déjà été déterminées dans la littérature [2]. Leurs valeurs sont rapportées dans le diagramme d'Arrhénius de la figure 5 où elles sont comparées aux vitesses déterminées pour ces deux produits dans l'air à l'état caoutchoutique (entre 250 et $320^{\circ} \mathrm{C}$ dans cette étude). Deux comportements distincts sont clairement mis en évidence de part et d'autre du point de fusion $\left(\mathrm{T}_{\mathrm{f}} \approx 343^{\circ} \mathrm{C}\right) \mathrm{du}$ PEEK. À l'état caoutchoutique, les deux produits de dégradation s'accumulent avec des vitesses assez proches alors que, à l'état fondu, leurs vitesses d'accumulation diffèrent sensiblement, cet écart grandissant avec l'élévation de la température. En plus d'un saut du facteur pré-exponentiel, principalement dû à la transformation de la phase cristalline en phase amorphe, on observe clairement une rupture de pente dans le graphe d'Arrhenius de $\mathrm{r}_{\mathrm{ox}}$ du benzoate de phényle au passage de $\mathrm{T}_{\mathrm{f}}$, alors que pour la fluorénone, la pente reste quasiment identique. Les énergies d'activation trouvées pour ces deux produits de dégradation de part et d'autre de $\mathrm{T}_{\mathrm{f}}$ sont rapportées dans le tableau 2 .

Une analyse plus attentive de la région des carbonyles montre une inversion dans les prédominances relatives des bandes d'absorption IR du benzoate de phényle $\left(\right.$ à $\left.1740 \mathrm{~cm}^{-1}\right)$ et de la fluorénone $\left(1718 \mathrm{~cm}^{-1}\right)$ au passage de $\mathrm{T}_{\mathrm{f}}$ (Fig. 6). Dans leur étude, Cole et Casella [2] avaient déjà montré une prédominance du benzoate de phényle sur la fluorénone à $400^{\circ} \mathrm{C}$ et le comportement inverse à plus haute température (typiquement entre 430 et $485^{\circ} \mathrm{C}$ ). Pour expliquer cette inversion de comportement, ils avaient avancé l'hypothèse d'un contrôle de l'oxydation par la diffusion d'oxygène dans des films de PEEK de $50 \mu \mathrm{m}$ d'épaisseur à partir de $430^{\circ} \mathrm{C}$. 

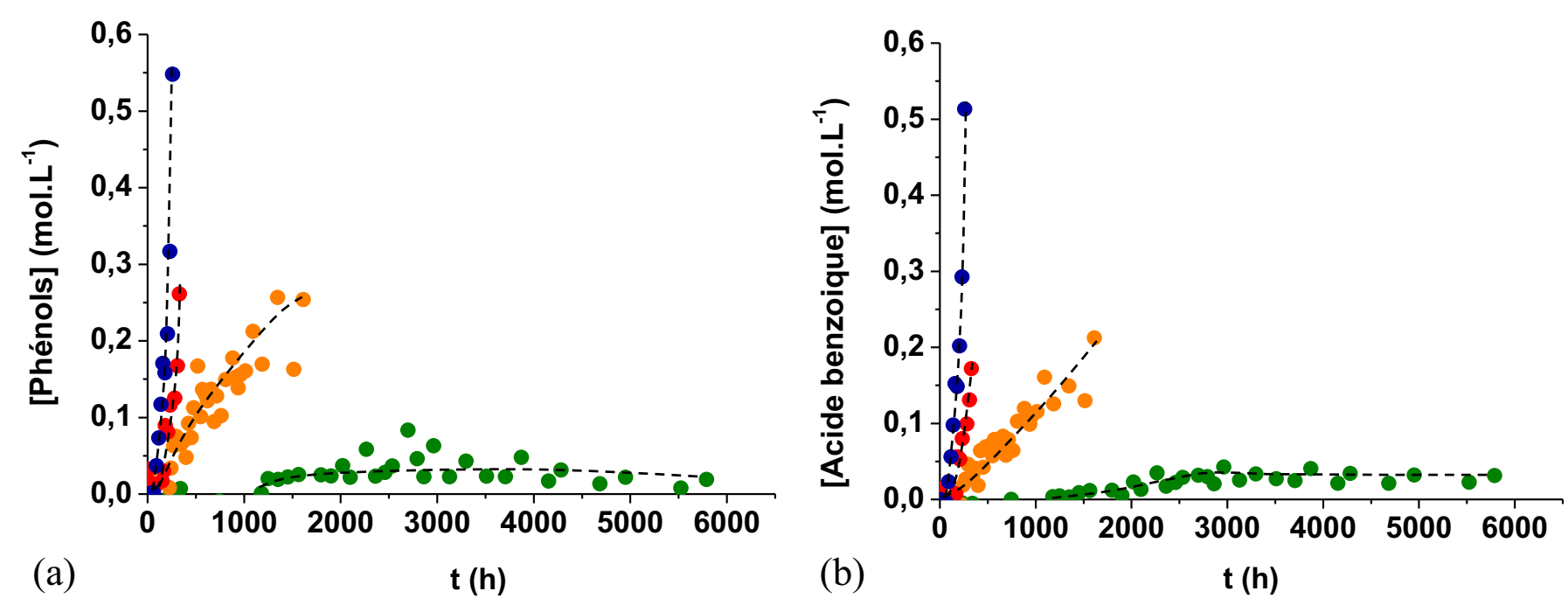

Fig. 4. Évolution des concentrations en phénols (a) et acide benzoïque (b) au cours du vieillissement thermique du PEEK dans l'air à 250 (en vert), 280 (orange), 300 (rouge) et $320^{\circ} \mathrm{C}$ (bleu).

Fig 4. Changes in the concentrations of phenols (a) and benzoic acid (b) during the thermal ageing of PEEK in air at 250 (in green), 280 (orange), 300 (red) and $320^{\circ} \mathrm{C}$ (blue).

Tableau 1. Énergie d'activation des temps d'induction $\left(t_{i}\right)$ et des vitesses maximales $\left(r_{o x}\right)$ de formation de l'anhydride benzoïque, du benzoate de phényle, de la fluorénone, des phénols et de l'acide benzoïque dans l'air entre 250 et $320^{\circ} \mathrm{C}$.

Table 1. Activation energy of the induction times $\left(t_{i}\right)$ and the maximum rates $\left(r_{o x}\right)$ of the formation of benzoic anhydride, phenyl benzoate, fluorenone, phenols and benzoic acid in air between 250 and $320^{\circ} \mathrm{C}$.

\begin{tabular}{lll}
\hline Produits & $\begin{array}{l}\mathrm{E}_{\mathrm{a}} \mathrm{de} \mathrm{t}_{\mathrm{i}} \\
(\mathrm{kJ.mol})\end{array}$ & $\begin{array}{l}\mathrm{E}_{\mathrm{a}} \mathrm{de} \mathrm{r}_{\mathrm{ox}} \\
(\mathrm{kJ.mol})\end{array}$ \\
dans l'air & dans l'air \\
\hline Anhydride benzoïque & 102 & 177 \\
Benzoate de phényle & 106 & 143 \\
Fluorénone & 134 & 166 \\
Phénols & 102 & 166 \\
Acide benzoïque & 92 & 175 \\
\hline
\end{tabular}

En revanche, à $400^{\circ} \mathrm{C}$, l'oxydation serait suffisamment lente pour permettre à l'oxygène de diffuser jusqu'au cœur des films. Le benzoate de phényle, qui est formé uniquement en présence d'oxygène, serait alors présent dans toute l'épaisseur des films et de ce fait, deviendrait le produit de dégradation majoritaire. Cependant, cette hypothèse ne permet pas d'expliquer la totale disparition du benzoate de phényle au-dessus du $\mathrm{T}_{\mathrm{f}}$.

Or, on sait que certaines réactions chimiques, en particulier les recombinaisons des espèces radicalaires, sont favorisées par l'augmentation de la mobilité moléculaire au passage d'une transition physique. Malheureusement, la littérature ne propose pas de mécanisme de formation du benzoate de phényle. Une possibilité, pourtant, serait la terminaison par couplage des radicaux carboxyle et

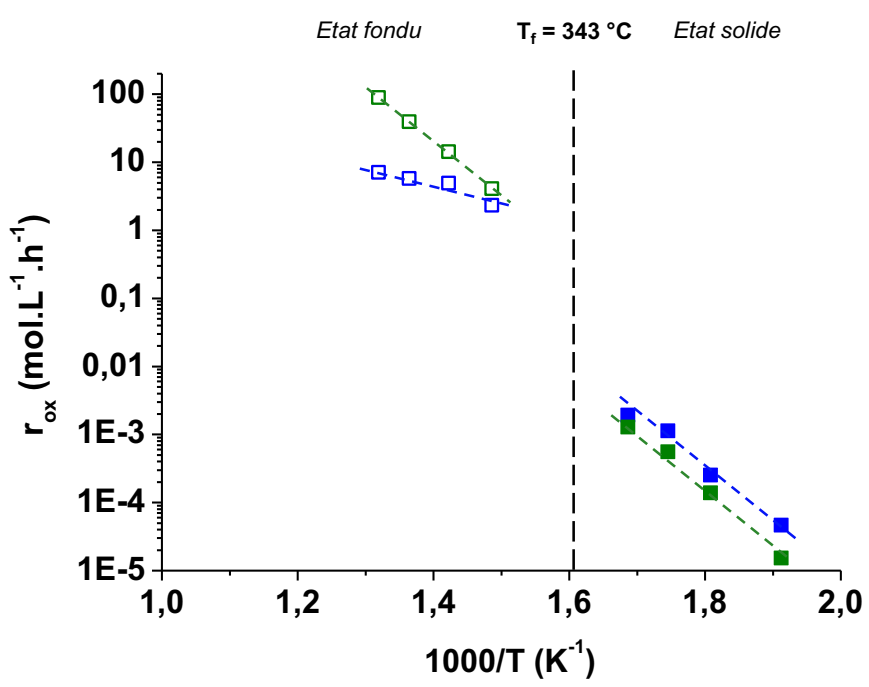

Fig. 5. Graphe d'Arrhenius des vitesse maximales $\left(\mathrm{r}_{\mathrm{ox}}\right)$ de formation du benzoate de phényle (en bleu) et de la fluorénone (vert) dans l'air entre 250 et $485^{\circ} \mathrm{C}$. Les résultats de cette étude (entre 250 et $320^{\circ} \mathrm{C}$ ) sont en symboles pleins alors que ceux de la littérature (entre 400 et $485^{\circ} \mathrm{C}$ [2]) sont en symboles vides.

Fig 5. Arrhenius diagram of the maximum rates $\left(r_{o x}\right)$ of the formation of phenyl benzoate (in blue) and fluorenone (green) in air between 250 and $485^{\circ} \mathrm{C}$. The results of the present study (between 250 and $320^{\circ} \mathrm{C}$ ) are given in full symbols whereas those of the literature (between 400 and $485^{\circ} \mathrm{C}$ [2]) are given in empty symbols.

phénoxyle (Fig. 7) dont les origines seront détaillées plus loin dans ce document. Cette réaction nécessite le réarrangement du radical phényle sous une forme quinonique. Bien entendu, elle est en compétition avec la diffusion de ces deux radicaux hors de la cage, qui va conduire à la formation d'une grande variété de produits de 
Tableau 2. Énergie d'activation des vitesses maximales $\left(\mathrm{r}_{\mathrm{ox}}\right)$ de formation du benzoate de phényle et de la fluorénone dans l'air à l'état caoutchoutique entre 250 et $320^{\circ} \mathrm{C}$ (cette étude) et à l'état fondu entre 400 et $485^{\circ} \mathrm{C}$ (littérature [2]).

Table 2. Activation energy of the maximum rates $\left(r_{o x}\right)$ of the formation of phenyl benzoate and fluorenone in air in rubbery state between 250 and $320^{\circ} \mathrm{C}$ (present study) and in molten state between 400 and $485^{\circ} \mathrm{C}$ (literature [2]).

\begin{tabular}{llc}
\hline \multirow{2}{*}{ Produits } & \multicolumn{2}{c}{$\mathrm{E}_{\mathrm{a}}$ de $\mathrm{r}_{\mathrm{ox}}\left(\mathrm{kJ} \cdot \mathrm{mol}^{-1}\right)$} \\
\cline { 2 - 3 } & $\begin{array}{l}\text { État } \\
\text { caoutchoutique }\end{array}$ & $\begin{array}{c}\text { État } \\
\text { fondu }\end{array}$ \\
\cline { 2 - 3 } Benzoate de phényle & 143 & 52 \\
Fluorénone & 166 & 153 \\
\hline
\end{tabular}

dégradation (acide benzoïque, dioxyde de carbone, phénols, etc.). On s'attend à ce que l'élévation de la température favorise la diffusion et ainsi, réduise sensiblement le rendement de formation du benzotate de phényle, comme précédemment observé sur la figure 5 .

Pour mettre en évidence l'effet de la pression partielle d'oxygène sur la cinétique d'oxydation du PEEK, des analyses IRTF ont été réalisées sur des films vieillis sous des pressions partielles d'oxygène comprises entre 0,21 (dans l'air) et 50 bars entre 180 et $250^{\circ} \mathrm{C}$. À titre d'exemple, les figures 8 et 9 rapportent l'évolution des concentrations des différents produits carbonyle (anhydride benzoïque, benzoate de phényle et fluorénone) et hydroxyle (phénols et acide benzoïque) au cours du vieillissement thermique du PEEK sous différentes pressions partielles d'oxygène à $250{ }^{\circ} \mathrm{C}$. Il apparaît clairement que la pression partielle d'oxygène accélère la cinétique d'oxydation du PEEK. L'anhydride benzoïque et le benzoate de phényle sont les deux produits de dégradation majoritaires sous forte pression partielle d'oxygène. Par contre, les phénols et l'acide benzoïque se forment en très faible concentration, quelle que soit la pression partielle d'oxygène.

A titre d'exemple, les valeurs de $t_{i}$ et $r_{o x}$ déterminées pour l'anhydride benzoïque, le benzoate de phényle et la fluorénone à $250^{\circ} \mathrm{C}$ ont été portées en fonction de la pression partielle d'oxygène sur la figure 10 . Si les valeurs de $t_{i}$ sont comparables, en revanche, les valeurs de $r_{o x}$ se rangent dans l'ordre d'importance des produits carbonyle rapporté précédemment (Fig. 8), à savoir :

benzoate de phényle > anhydride benzoïque >> fluorénone

On constate que les courbes présentent une allure hyperbolique et atteignent un plateau de saturation qui est caractéristique du régime en excès d'oxygène à forte pression partielle d'oxygène. En d'autres termes, $t_{i}$ et $r_{o x}$ deviennent presque indépendants de la pression partielle d'oxygène au-dessus d'une valeur critique, notée $P_{c}$.

Les valeurs de $\mathrm{P}_{\mathrm{c}}$, ainsi que les valeurs de $t_{i}$ et $\mathrm{r}_{\mathrm{ox}}$ en excès d'oxygène, déterminées à 220 et $250^{\circ} \mathrm{C}$ sont récapitulées au tableau 3. Notons que l'excès d'oxygène n'est pas encore atteint sous 50 bars d'oxygène à 180 et $200^{\circ} \mathrm{C}$. Néanmoins, il apparaît clairement que $\mathrm{P}_{\mathrm{c}}$ est une fonction décroissante de la température. Ainsi, entre 180 et $250{ }^{\circ} \mathrm{C}$, tous les vieillissements effectués dans l'air correspondent à un régime en défaut d'oxygène.

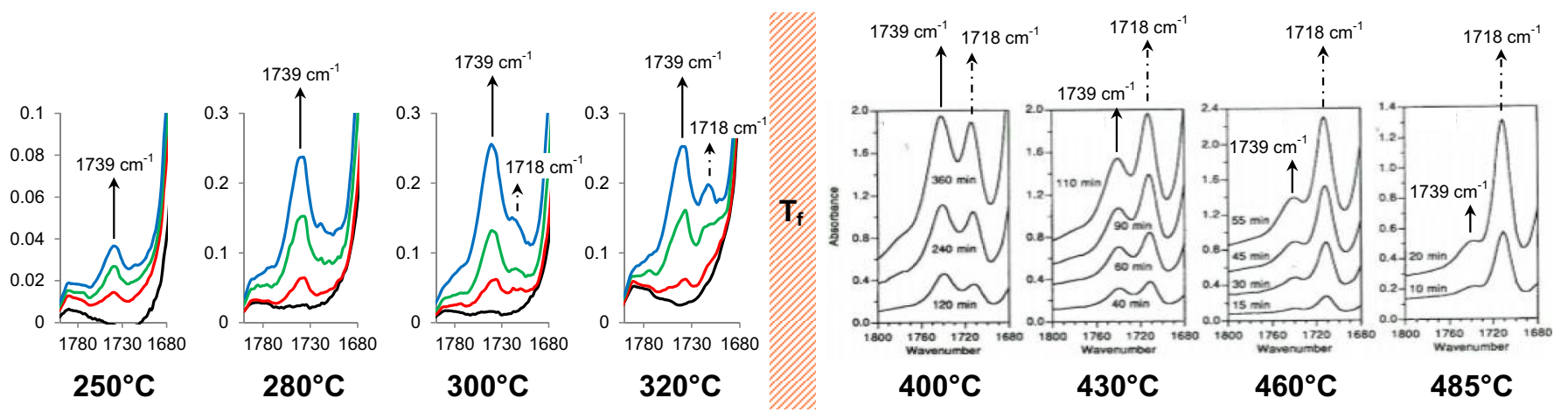

Fig. 6. Prédominance relative des bandes d'absorption IR du benzoate de phényle (à $\left.1740 \mathrm{~cm}^{-1}\right)$ et de la fluorénone $\left(1718 \mathrm{~cm}^{-1}\right)$ au cours du vieillissement thermique du PEEK dans l'air à l'état caoutchoutique entre 250 et $320^{\circ} \mathrm{C}$ (cette étude) et à l'état fondu entre 400 et $485^{\circ} \mathrm{C}$ (littérature [2]).

Fig 6. Relative predominance of the IR absorption bands of phenyl benzoate (at $\left.1740 \mathrm{~cm}^{-1}\right)$ and fluorenone $\left(1718 \mathrm{~cm}^{-1}\right)$ during the thermal ageing of PEEK in air in rubbery state between 250 and $320^{\circ} \mathrm{C}$ (present study) and in molten state between 400 and $485^{\circ} \mathrm{C}($ literature [2]).

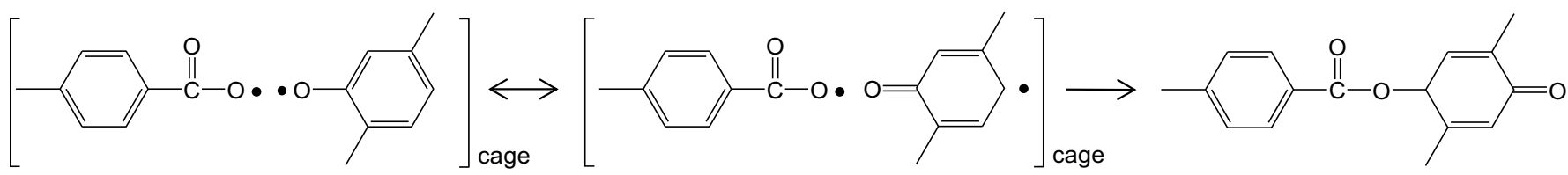

Fig. 7. Mécanisme de formation du benzoate de phényle par couplage des radicaux carboxyle et phénoxyle.

Fig 7. Formation mechanism of phenyl benzoate by coupling carboxyl and phenoxyl radicals. 

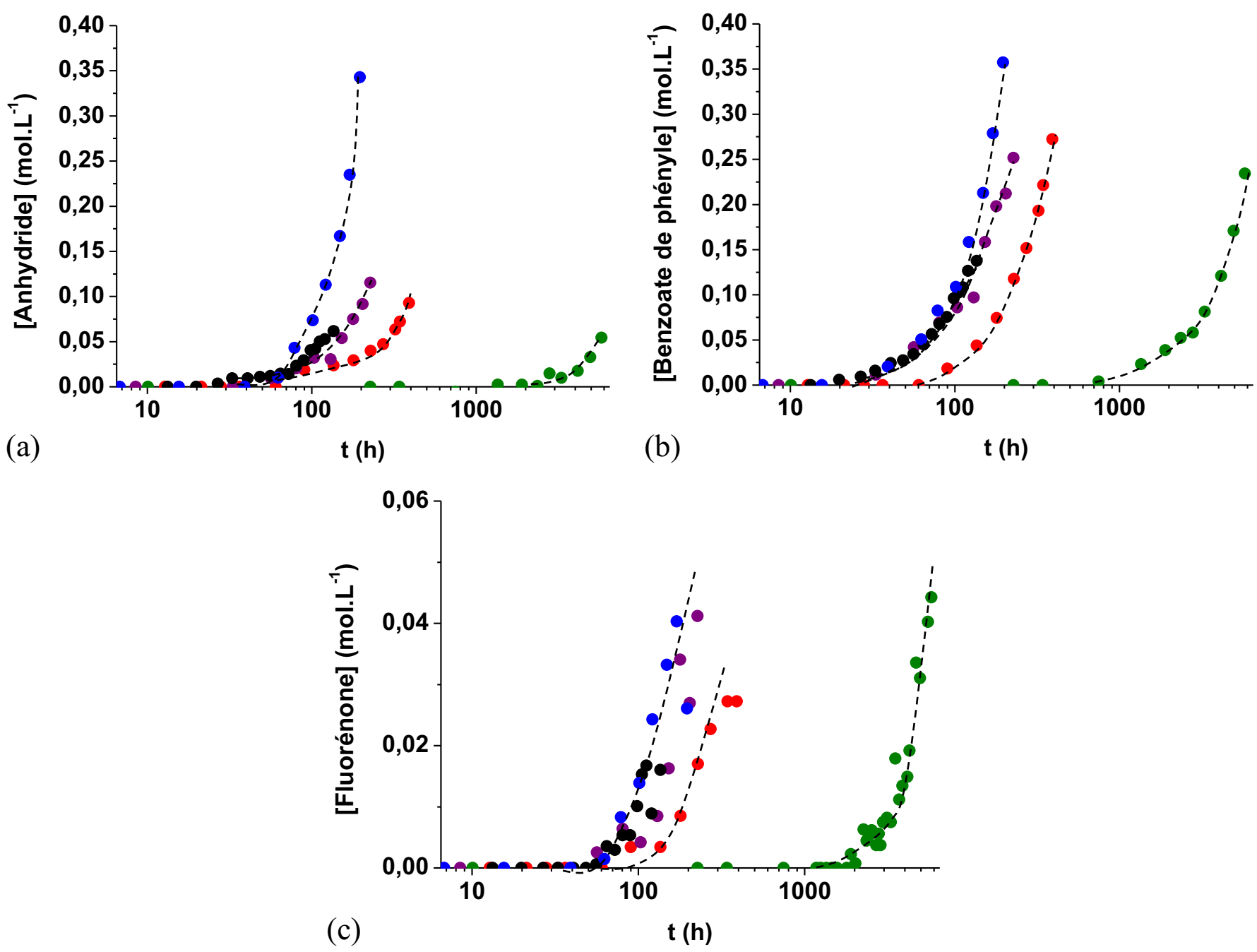

Fig. 8. Évolution des concentrations en anhydride benzoïque (a), benzoate de phényle (b) et fluorénone (c) au cours du vieillissement thermique du PEEK sous une pression de 0,21 bar (en vert), 3 bars (rouge), 6 bars (violet), 8 bars (noir) et 10 bars d'oxygène (bleu) à $250^{\circ} \mathrm{C}$.

Fig 8. Changes in the concentrations of benzoic anhydride (a), phenyl benzoate (b) and fluorenone (c) during the thermal ageing of PEEK under an oxygen pressure of 0.21 bar (in green), 3 bars (red), 6 bars (violet), 8 bars (black) and 10 bars (blue) at $250^{\circ} \mathrm{C}$.

L'ensemble de ces résultats expérimentaux permet de proposer un mécanisme de dégradation thermique du PEEK. Comme déjà proposé dans la littérature [2-4], ce mécanisme est amorcé par la rupture des liaisons cétone et éther de l'unité monomère et ainsi, conduit à la formation de trois principaux types d'espèce radicalaire (Fig. 11): acyle $\left(\mathrm{P}_{1}^{\bullet}\right)$, phénoxyle $\left(\mathrm{P}_{2}^{\bullet}\right)$ et phényle $\left(\mathrm{P}_{3} \bullet\right.$ ou $\left.\mathrm{P}_{4} \bullet\right)$. Tout d'abord, les radicaux acyles sont à l'origine de la formation du monoxyde de carbone $(\mathrm{CO})$ par décarbonylation, et du benzaldéhyde par arrachement d'hydrogène sur les cycles aromatiques. En raison de la faible énergie de dissociation de la liaison $\mathrm{C}-\mathrm{H}$ dans un groupe aldéhyde $\left(\mathrm{E}_{\mathrm{D}}=375 \mathrm{~kJ} . \mathrm{mol}^{-1}[20]\right.$, contre $\mathrm{E}_{\mathrm{D}}=393 \mathrm{~kJ} \cdot \mathrm{mol}^{-1}$ dans un groupe méthylène), on s'attend à ce que le benzaldéhyde s'oxyde quasi-instantanément en acide benzoïque. Il n'est donc pas étonnant que ce produit carbonyle ne soit pas détecté par spectoscopie IRTF [21]. En revanche, les radicaux phénoxyle conduisent à la formation de phénols par arrachement d'hydrogène sur les cycles aromatiques.
Comme les stabilisants phénoliques [22], ils peuvent aussi être stabilisés par résonance et entraîner la formation d'espèces quinoniques.

Ainsi, la propagation de l'oxydation s'effectue en deux étapes. D'abord, la formation de radicaux peroxy par addition d'oxygène sur les radicaux acyle et phényle. Ensuite, la formation d'hydroperoxydes (POOH) par arrachement d'hydrogène sur les cycles aromatiques par les radicaux peroxy (Fig. 12). Rappelons que, à haute température (typiquement pour $\mathrm{T}>200^{\circ} \mathrm{C}$ ), la décomposition des hydroperoxydes est exclusivement unimoléculaire [23].

Les hydroperoxydes issus de l'oxydation des radicaux acyle sont à l'origine de la formation de l'acide benzoïque. En effet, leur décomposition génère des radicaux carbonyle qui peuvent soit se recombiner par arrachement d'hydrogène sur les cycles aromatiques et ainsi, donner l'acide benzoïque, soit se réarranger pour former du dioxyde de carbone $\left(\mathrm{CO}_{2}\right)$ par décarboxylation (Fig. 13). Quant aux phénols, ils proviennent des radicaux phénoxyle générés 

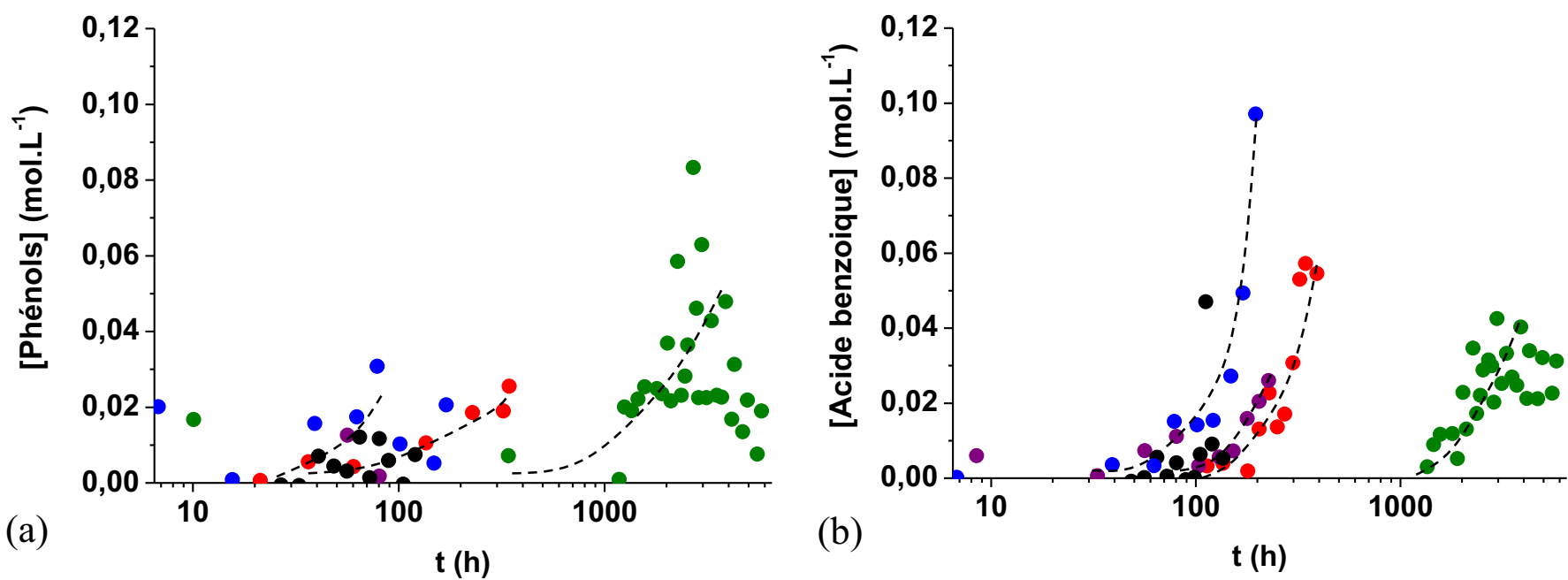

Fig. 9. Évolution des concentrations en phénols (a) et acide benzoïque (b) au cours du vieillissement thermique du PEEK sous une pression de 0,21 bar (en vert), 3 bars (rouge), 6 bars (violet), 8 bars (noir) et 10 bars d'oxygène (bleu) à $250^{\circ} \mathrm{C}$.

Fig 9. Changes in the concentrations of phenols (a) and benzoic acid (b) during the thermal ageing of PEEK under an oxygen pressure of 0.21 bar (in green), 3 bars (red), 6 bars (violet), 8 bars (black) and 10 bars (blue) at $250^{\circ} \mathrm{C}$.
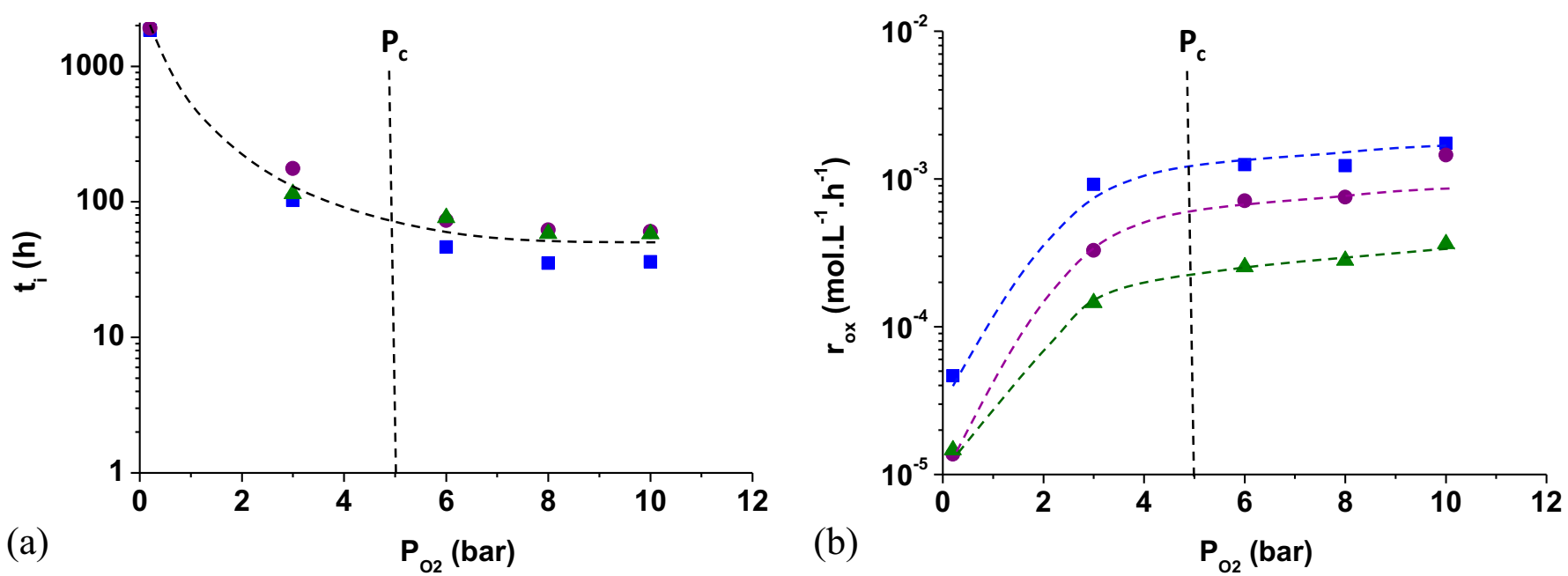

Fig. 10. Variation des temps d'induction (a) et des vitesses maximales (b) de formation de l'anhydride benzoïque ( $\bigcirc)$, du benzoate de phényle $(\square)$ et de la fluorénone $(\Lambda)$ avec la pression partielle d'oxygène à $250^{\circ} \mathrm{C}$.

Fig 10. Changes of the induction times (a) and the maximum rates (b) of the formation of benzoic anhydride (O), phenyl benzoate and fluorenone (\) with the oxygen partial pressure at $250^{\circ} \mathrm{C}$.

par la thermolyse (Fig. 11), mais aussi par la décomposition des hydroperoxydes issus de l'oxydation des radicaux phényle (Fig. 14).

Comme déjà proposé dans la littérature [2,6], la réaction de terminaison par couplage intramoléculaire de radicaux phényle permet d'expliquer la formation de la fluorénone en défaut d'oxygène (Fig. 15). Cependant, ce produit de dégradation est également présent, bien qu'en plus faible quantité, sous forte pression partielle d'oxygène. Une seconde réaction de terminaison, par couplage intramoléculaire de radicaux peroxy a donc été proposée dans cette étude pour rendre compte de ce comportement (Fig. 16).

\subsection{Modifications de la structure macromoléculaire}

L'évolution des masses moléculaires pendant le vieillissement des polymères permet de mettre en évidence les modifications au niveau de l'architecture macromoléculaire, c'est-à-dire les coupures de chaîne (notées S) et les actes de réticulation (notés X). Comme chaque coupure de chaîne fait apparaître une nouvelle chaîne tandis que chaque acte de réticulation fait disparaître une chaîne, on peut écrire:

$$
S-X=\frac{1}{M_{n}}-\frac{1}{M_{n 0}},
$$


Tableau 3. Pression critique $\left(\mathrm{P}_{\mathrm{c}}\right)$, temps d'induction $\left(\mathrm{t}_{\mathrm{i}}\right)$ et vitesse maximale $\left(\mathrm{r}_{\mathrm{ox}}\right)$ de formation de l'anhydride benzoïque, du benzoate de phényle et de la fluorénone en excès d'oxygène à 220 et $250^{\circ} \mathrm{C}$.

Table 3. Critical pressure $\left(P_{C}\right)$, induction time $\left(t_{i}\right)$ and maximum rate $\left(r_{o x}\right)$ of the formation of benzoic anhydride, phenyl benzoate and fluorenone in oxygen excess at 220 and $250^{\circ} \mathrm{C}$.

\begin{tabular}{lllll}
\hline Produits & $\mathrm{T}\left({ }^{\circ} \mathrm{C}\right)$ & $\mathrm{P}_{\mathrm{c}}$ (bars) & $\mathrm{t}_{\mathrm{i}}$ en excès d'O $\mathrm{O}_{2}(\mathrm{~h})$ & $\mathrm{r}_{\mathrm{ox}}$ en excès d' $\mathrm{O}_{2}\left(\mathrm{~mol}^{2} \mathrm{~L}^{-1} \cdot \mathrm{h}^{-1}\right)$ \\
\hline Anhydride & & 227 & $8,2.10^{-4}$ \\
Benzoate de phényle & 220 & 20 & 262 & $8,4.10^{-4}$ \\
Fluorénone & & 250 & $0,97.10^{-4}$ \\
Anhydride & & 62 & $19,7.10^{-4}$ \\
Benzoate de phényle & 250 & 5 & 39 & $13,1.10^{-4}$ \\
Fluorénone & & 64 & $4,3.10^{-4}$ \\
\hline
\end{tabular}

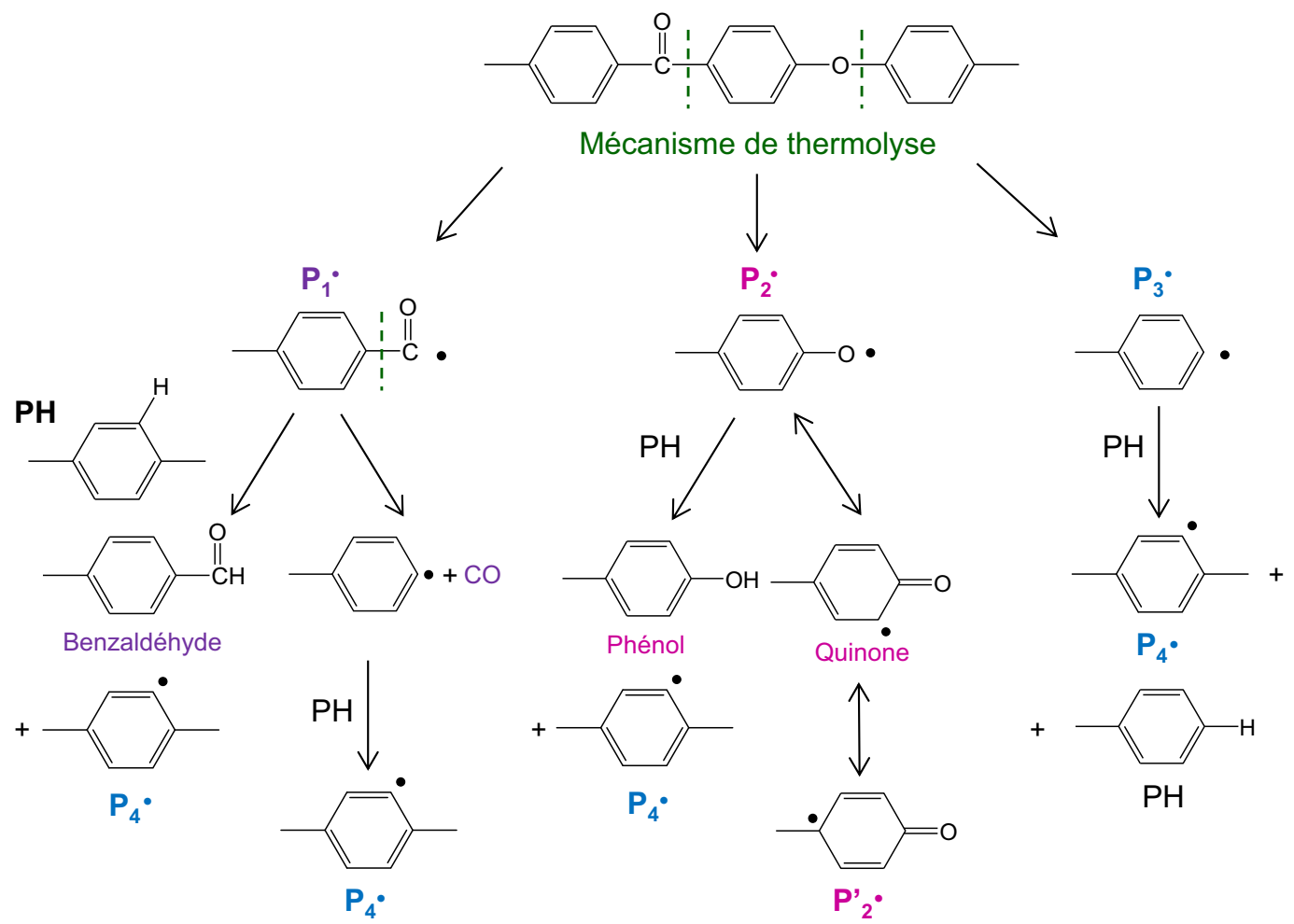

Fig. 11. Amorçage de la dégradation thermique du PEEK par rupture thermolytique de liaisons cétone et éther de la chaîne macromoléculaire. Formation de plusieurs espèces radicalaires notées : $\mathrm{P}_{1} \bullet, \mathrm{P}_{2} \bullet, \mathrm{P}_{3} \bullet$ et $\mathrm{P}_{4} \bullet$.

Fig 11. Initiation of the thermal degradation of PEEK by thermolytic breakdown of ketone and ether bonds of the macromolecular chain. Formation of several radical species denoted: $P_{1}^{\bullet}, P_{2}^{\bullet}, P_{3}^{\bullet}$ and $P_{4}^{\bullet}$.

où $M_{n 0}$ et $M_{n}$ sont respectivement les masses moléculaires moyennes en nombre avant et après vieillissement.

Cependant, il n'a pas été possible de suivre l'évolution de la masse moléculaire du PEEK pendant son vieillissement thermique par des méthodes d'analyse directes, de type chromatographique (CES ou CPG) ou viscosimétrique (en solution), car ce polymère n'est pas soluble dans les solvants usuels (acétone, toluène, acétate d'éthyle, etc.). Nous avons donc eu recours à la calorimétrie différentielle (DSC) qui donne accès de manière indirecte à la valeur de $M_{n}$ connaissant la valeur de $T_{g}$ via la relation de Fox-Flory [24]:

$$
T_{g}-T_{g 0}=-k_{F F}\left(\frac{1}{M_{n}}-\frac{1}{M_{n 0}}\right),
$$

où $\mathrm{k}_{\mathrm{FF}}$ est la constante de Fox-Flory (exprimée en K.mol.kg ${ }^{-1}$ ) qui augmente avec la rigidité des chaînes.

La valeur de $\mathrm{k}_{\mathrm{FF}}$ a été déterminée à partir des températures de transition vitreuse et des masses moléculaires de plusieurs 


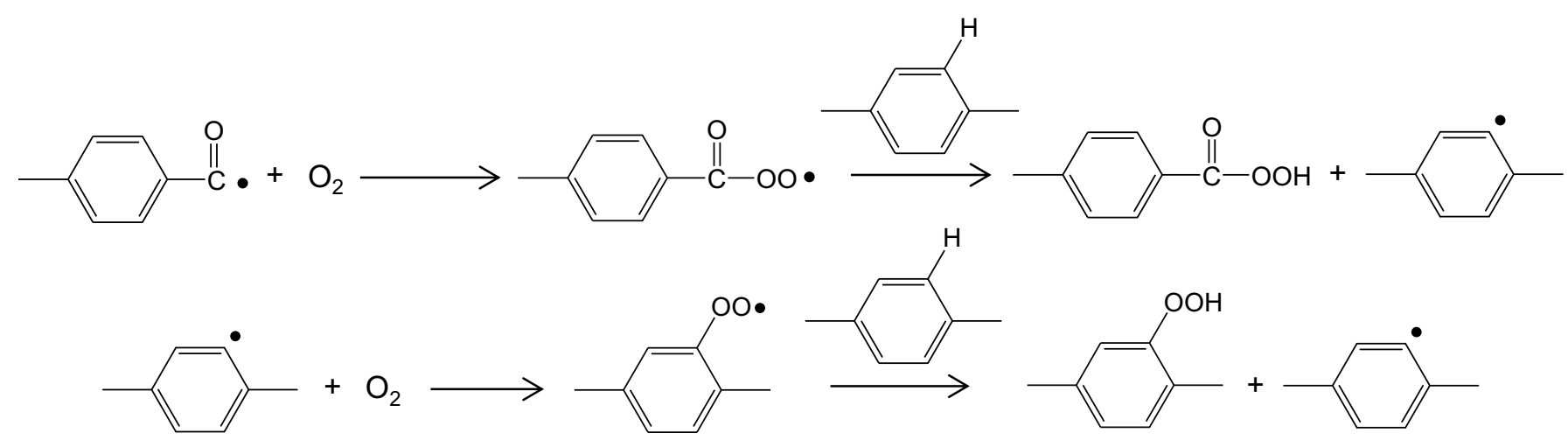

Fig. 12. Addition d'oxygène sur les radicaux acyle et phényle suivie de la formation d'hydroperoxydes par arrachement d'hydrogène sur les cycles aromatiques.

Fig 12. Oxygen addition onto acyl and phenyl radicals followed by the formation of hydroperoxides by hydrogen abstraction on aromatic rings.

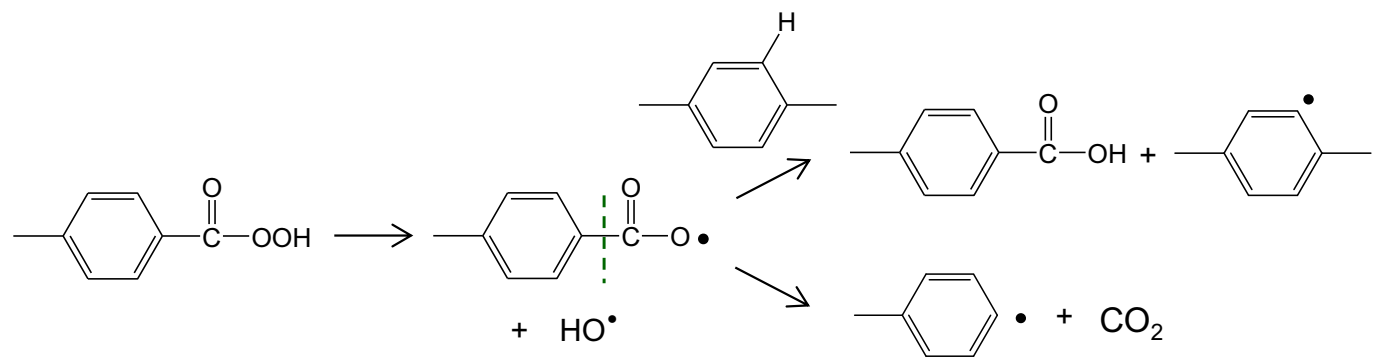

Fig. 13. Amorçage de la dégradation thermique par décomposition unimoléculaire de l'hydroperoxyde issu du radical acyle, entraînant la formation de l'acide benzoïque (en haut) ou une décarboxylation (en bas).

Fig 13. Initiation of the thermal degradation by unimolecular decomposition of the hydroperoxide resulting from the acyl radical. Formation of benzoic acid (top) or decarboxylation (below).
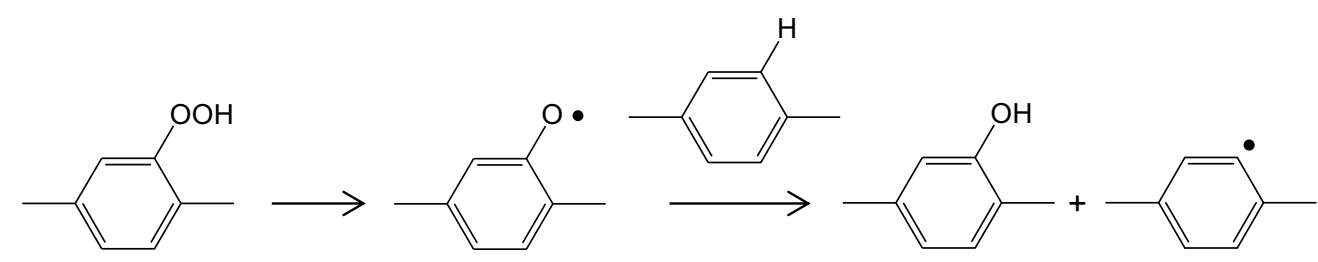

$+\mathrm{HO}^{\bullet}$

Fig. 14. Amorçage de la dégradation thermique par décomposition unimoléculaire de l'hydroperoxyde issu du radical phényle. Formation d'un phénol.

Fig 14. Initiation of the thermal degradation by unimolecular decomposition of the hydroperoxide resulting from the phenyl radical. Formation of phenol.

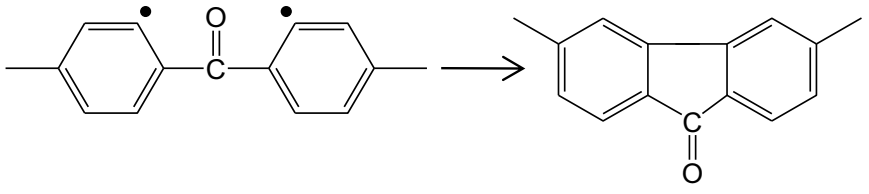

Fig. 15. Mécanisme de formation de la fluorénone par couplage intramoléculaire des radicaux phényle.

Fig 15. Formation mechanism of fluorenone by intramolecular coupling of phenyl radicals. grades de PEEK Victrex. En effet, la pente de la courbe $\mathrm{T}_{\mathrm{g}}=\mathrm{f}\left(1 / \mathrm{M}_{\mathrm{n}}\right)$ donne accès à la valeur $\operatorname{dek}_{\mathrm{FF}}$ tandis que l'ordonnée à l'origine correspond à la valeur de $\mathrm{T}_{\mathrm{g}}{ }^{\infty}$ (c'est-àdire à la $\mathrm{T}_{\mathrm{g}}$ théorique du polymère linéaire de même structure chimique mais de masse moléculaire infinie). Ainsi, pour le PEEK Victrex, on a trouvé : $\mathrm{k}_{\mathrm{FF}}=204 \mathrm{~K} . \mathrm{kg} \cdot \mathrm{mol}^{-1}$ et $\mathrm{T}_{\mathrm{g}}{ }^{\infty}=$ $432 \mathrm{~K}$. Notons que cette dernière valeur est très proche de la $\mathrm{T}_{\mathrm{g}}{ }^{\infty}$ théorique $\left(\mathrm{T}_{\mathrm{g}}{ }^{\infty}=433 \mathrm{~K}\right)$ calculée à l'aide de la loi d'additivité molaire proposée par Van Krevelen dans son livre [25]. 


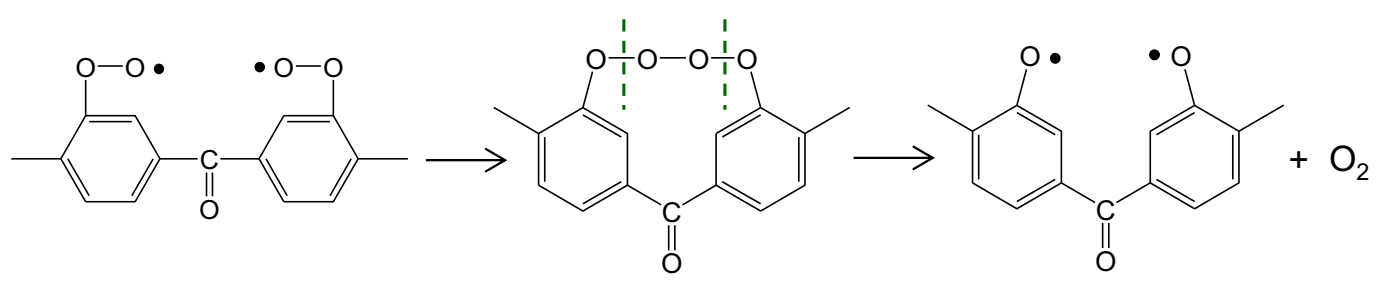<smiles>CCC1=CC=C(C(=O)C2=CC=C(C)C(=O)[CH2+]2)C(=O)C1C</smiles>

Fig. 16. Mécanisme de formation de la fluorénone par couplage intramoléculaire de radicaux peroxy.

Fig 16. Formation mechanism of fluorenone by intramolecular coupling of peroxy radicals.
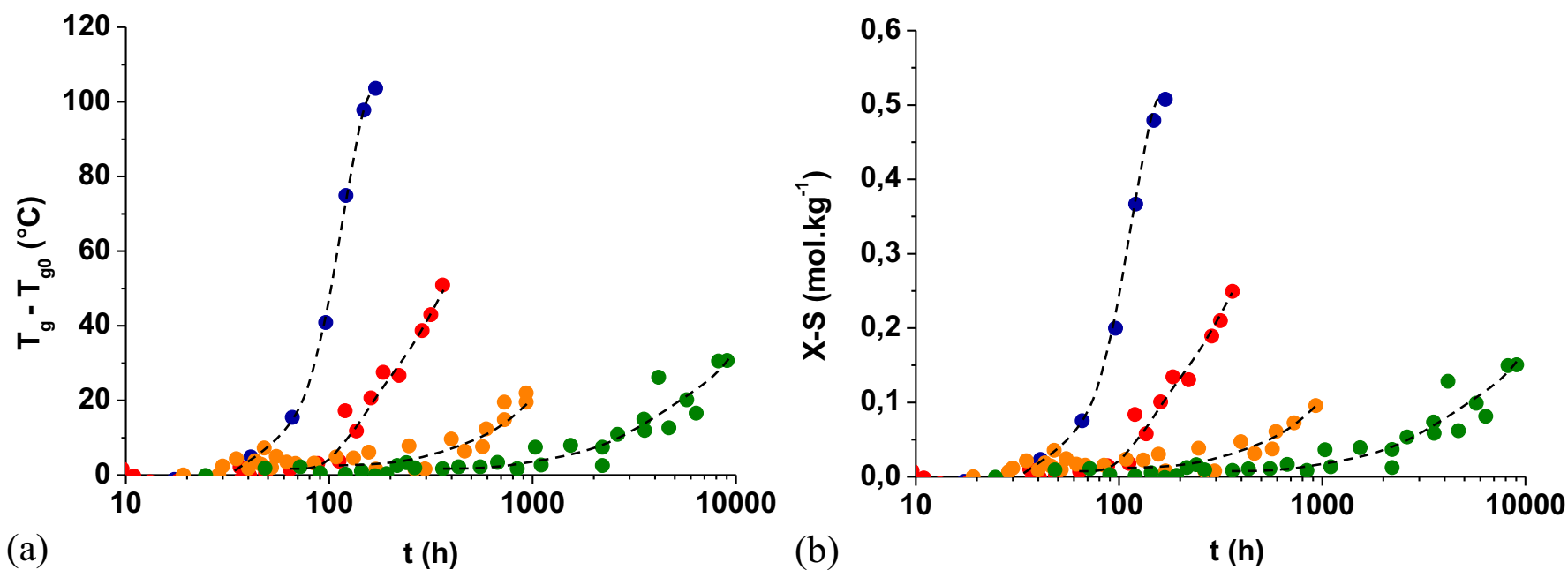

Fig. 17. Évolution des écarts entre la température de transition vitreuse et sa valeur initiale $\left(\mathrm{T}_{\mathrm{g}}-\mathrm{T}_{\mathrm{g} 0}\right)$ (a) et entre les nombres d'actes de réticulation et de coupure de chaîne $(\mathrm{X}-\mathrm{S})(\mathrm{b})$ au cours du vieillissement thermique du PEEK dans l'air à 250 (en vert), 280 (orange), 300 (rouge) et $320^{\circ} \mathrm{C}$ (bleu).

Fig 17. Changes in the gaps between the glass transition temperature and its initial value $\left(T_{g}-T_{g 0}\right)$ (a) and between the numbers of crosslinking and chain scission events $(X-S)(b)$ during the thermal ageing of PEEK in air at 250 (in green), 280 (orange), 300 (red) and $320^{\circ} \mathrm{C}($ blue).

En combinant les équations (7) et (8), on obtient la relation suivante entre $\mathrm{T}_{\mathrm{g}}$ et les nombres d'actes de coupure de chaîne et de réticulation :

$$
T_{g}-T_{g 0}=k_{F F}(X-S) .
$$

On voit que $\mathrm{T}_{\mathrm{g}}$ diminue avec le nombre de coupures de chaîne, mais augmente avec le nombre d'actes de réticulation.

La figure 17 rapporte l'évolution des écarts entre la $T_{g}$ et sa valeur initiale $\left(\mathrm{T}_{\mathrm{g}}-\mathrm{T}_{\mathrm{g} 0}\right)$, et entre les nombres d'actes de réticulation et de coupure de chaîne $(\mathrm{X}-\mathrm{S})$ des films de PEEK dans l'air entre 250 et $320^{\circ} \mathrm{C}$. Il apparaît clairement que la réticulation prédomine largement sur les coupures de chaîne à l'état caoutchoutique. Rappelons que cette prédominance relative avait déjà été observée par plusieurs auteurs à l'état fondu [3-5,7-9]. De plus, la réticulation est fortement thermo-activée.
Les valeurs $\mathrm{du}$ temps d'induction $\left(\mathrm{t}_{\mathrm{i}}\right)$ et la vitesse maximale de réticulation $\left(\mathrm{r}_{\mathrm{x}}\right)$ ont été déterminées graphiquement pour l'ensemble de ces courbes cinétiques. En première approche, les coupures de chaîne ont été négligées devant la réticulation, ce qui a permis de réécrire l'équation (9) sous la forme:

$$
r_{x}=\frac{d X}{\mathrm{~d} t}=\frac{1}{k_{F F}} \times \frac{d T_{g}}{\mathrm{~d} t} .
$$

Ces deux indicateurs obéissent à une loi d'Arrhenius entre 250 et $320^{\circ} \mathrm{C}$. Les valeurs d'énergie d'activation sont rapportées au tableau 4 où elles sont comparées aux valeurs obtenues pour les produits carbonyle. Elles sont toutes du même ordre de grandeur.

Des analyses DSC ont également été réalisées sur des films de PEEK après vieillissement sous différentes pression partielle d'oxygène comprises entre 0,21 et 20 bars à $250^{\circ} \mathrm{C}$. 
Tableau 4. Énergie d'activation du temps d'induction $\left(t_{i}\right)$ et de la vitesse maximale (r) des modifications moléculaires et macromoléculaires dans l'air entre 250 et $320^{\circ} \mathrm{C}$.

Table 4. Activation energy of induction time $\left(t_{i}\right)$ and maximum rate $(r)$ of molar and macromolecular modifications in air between 250 and $320^{\circ} \mathrm{C}$.

\begin{tabular}{lll}
\hline & $\begin{array}{l}\mathrm{E}_{\mathrm{a}} \text { de } \mathrm{t}_{\mathrm{i}} \\
(\mathrm{kJ.mol}) \\
\text { dans l'air }\end{array}$ & $\begin{array}{l}\mathrm{E}_{\mathrm{a}} \text { de r } \\
\left(\mathrm{kJ} . \mathrm{kol}^{-1}\right) \\
\text { dans l'air }\end{array}$ \\
\hline Anhydride benzoïque & 102 & 177 \\
Benzoate de phényle & 106 & 143 \\
Fluorénone & 134 & 166 \\
Réticulation & 113 & 220 \\
\hline
\end{tabular}

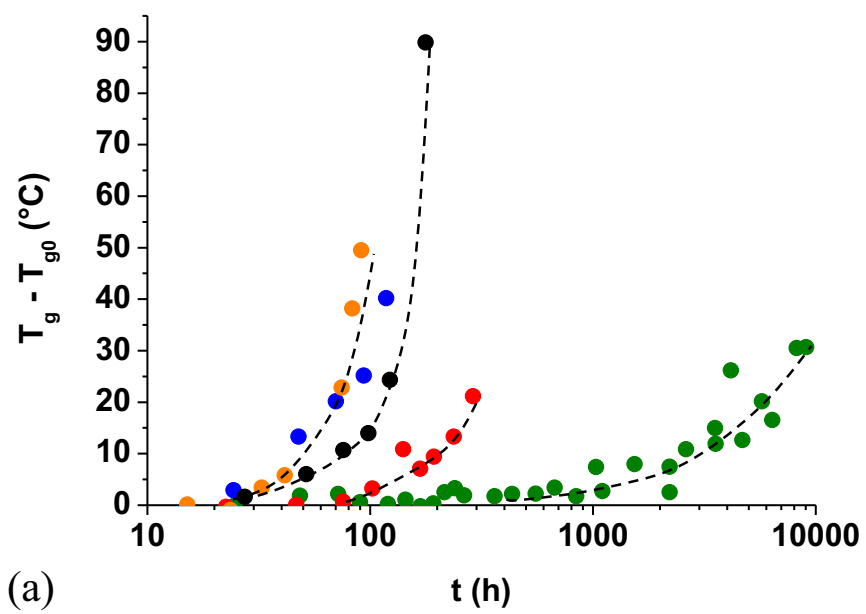

Les évolutions de $\left(\mathrm{T}_{\mathrm{g}}-\mathrm{T}_{\mathrm{g} 0}\right)$ et de $(\mathrm{X}-\mathrm{S})$ sont données sur la figure 18. On retrouve les mêmes comportements que dans l'air. D'une manière générale, la pression partielle d'oxygène accélère la cinétique de réticulation jusqu'à une pression critique de l'ordre de 5 bars à $250^{\circ} \mathrm{C}$, qui avait déjà été déterminée par spectroscopie IRTF.

Les valeurs de $\mathrm{t}_{\mathrm{i}}$ et $\mathrm{r}_{\mathrm{x}}$ déterminées à $250^{\circ} \mathrm{C}$ ont été portées en fonction de la pression partielle d'oxygène sur la figure 19. On constate que les courbes présentent une allure hyperbolique et atteignent un plateau caractéristique du régime d'excès d'oxygène à partir de $\mathrm{P}_{\mathrm{c}}=5$ bars. Les valeurs de $\mathrm{t}_{\mathrm{i}}$ et $\mathrm{r}_{\mathrm{x}}$ en excès d'oxygène à $250^{\circ} \mathrm{C}$ sont récapitulées au tableau 5 où elles sont comparées aux valeurs obtenues pour les produits carbonyle. Elles sont toutes du même ordre de grandeur. On en déduit que la réticulation est une conséquence directe de l'oxydation du PEEK.

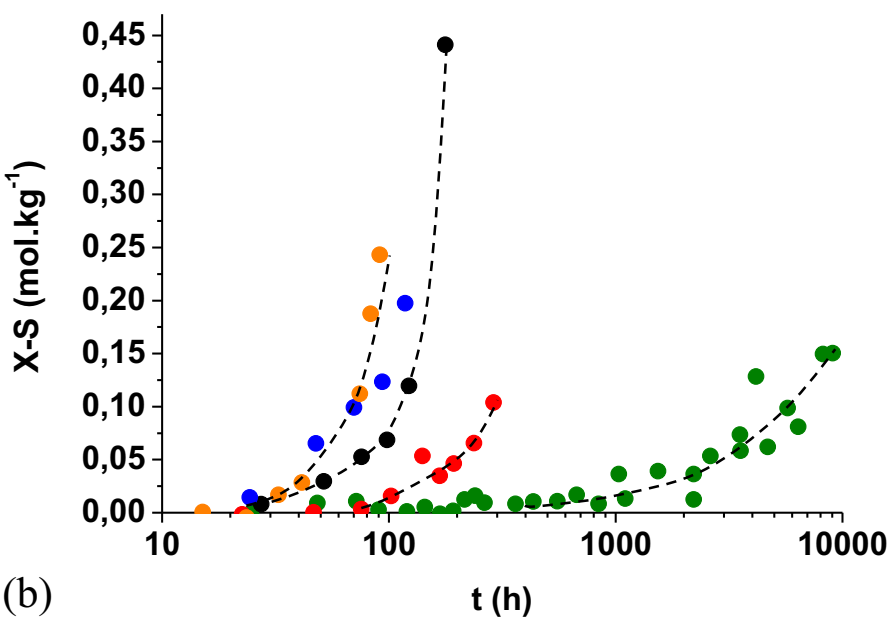

Fig. 18. Évolution des écarts entre la température de transition vitreuse et sa valeur initiale $\left(\mathrm{T}_{\mathrm{g}}-\mathrm{T}_{\mathrm{g} 0}\right)$ (a) et entre le nombre d'actes de réticulation et de coupure de chaîne $(\mathrm{X}-\mathrm{S})(\mathrm{b})$ au cours du vieillissement thermique du PEEK sous une pression de 0,21 bar (en vert), 3 bars (rouge), 8 bars (noir), 10 bars (bleu) et 20 bars d'oxygène (orange) à $250^{\circ} \mathrm{C}$.

Fig 18. Changes in the gaps between the glass transition temperature and its initial value (Tg-Tg0) (a) and the number of crosslinking and chain scission events $(X-S)(b)$ during the thermal ageing of PEEK under an oxygen pressure of 0.21 bar (in green), 3 bars (red), 8 bars (black), 10 bars (blue) and 20 bars (orange) at $250^{\circ} \mathrm{C}$.
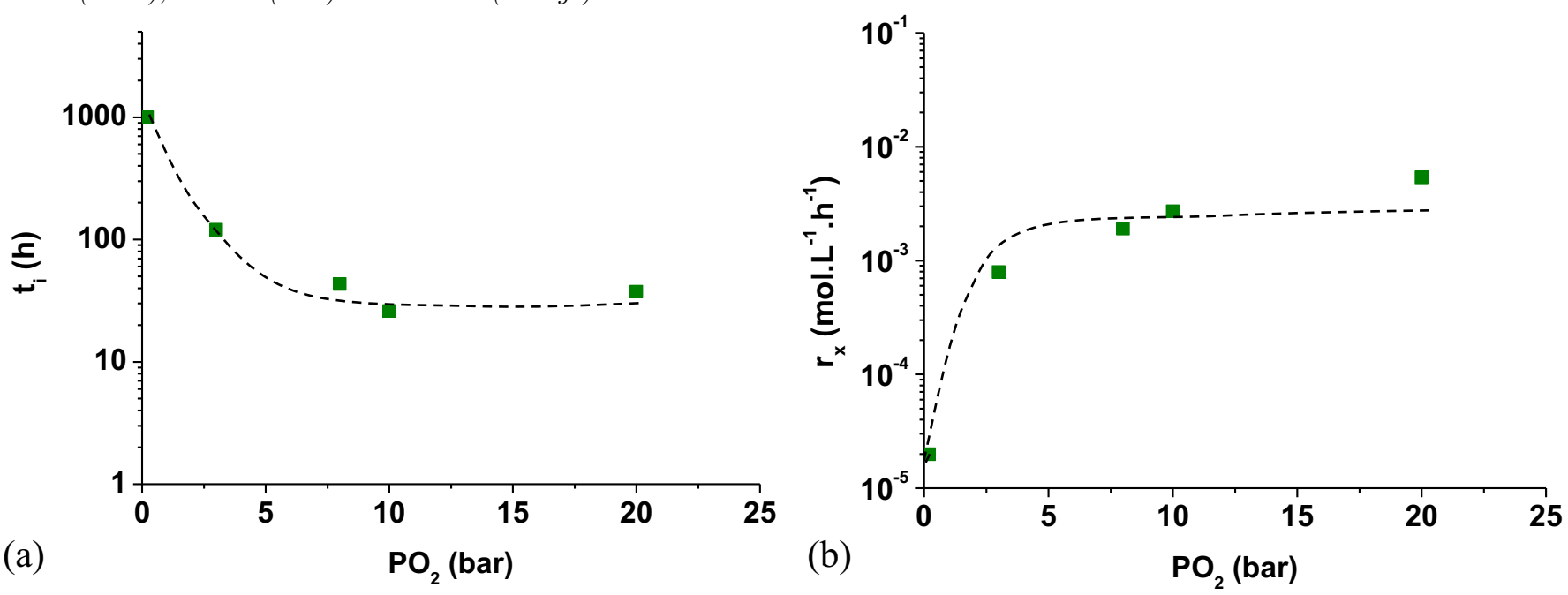

Fig. 19. Variation du temps d'induction (a) et de la vitesse maximale (b) de réticulation avec la pression partielle d'oxygène à $250^{\circ} \mathrm{C}$.

Fig 19. Changes in the induction time (a) and the maximum rate (b) of crosslinking with oxygen partial pressure at $250^{\circ} \mathrm{C}$. 
Comme déjà proposé dans la littérature [3-6], la terminaison par couplage intermoléculaire des radicaux phényle est le principal mécanisme de réticulation en défaut d'oxygène (Fig. 20). Cependant, cette réaction ne peut pas expliquer la réticulation du PEEK en excès

Tableau 5. Pression critique $\left(\mathrm{P}_{\mathrm{c}}\right)$, temps d'induction $\left(\mathrm{t}_{\mathrm{i}}\right)$ et vitesse maximale $(\mathrm{r})$ des modifications moléculaires et macromoléculaires en excès d'oxygène à $250^{\circ} \mathrm{C}$.

Table 5. Critical pressure $\left(P_{C}\right)$, induction time $\left(t_{i}\right)$ and maximum rate $(r)$ of molar and macromolecular modifications in oxygen excess at $250^{\circ} \mathrm{C}$.

\begin{tabular}{llll}
\hline & $\begin{array}{l}\mathrm{P}_{\mathrm{C}} \\
(\text { bars })\end{array}$ & $\begin{array}{l}\mathrm{t}_{\mathrm{i}} \text { en } \\
\text { excès } \\
\mathrm{d}^{\prime} \mathrm{O}_{2}(\mathrm{~h})\end{array}$ & $\begin{array}{l}\mathrm{r} \text { en } \\
\text { excès d'O } \\
\left(\mathrm{mol}_{2} \mathrm{~L}^{-1} \cdot \mathrm{h}^{-1}\right)\end{array}$ \\
\hline Réticulation & 5 & 36 & $33,3 \cdot 10^{-4}$ \\
Anhydride benzoïque & 5 & 65 & $19,7.10^{-4}$ \\
Benzoate de phényle & 5 & 30 & $13,1.10^{-4}$ \\
Fluorénone & 5 & 30 & $13,1.10^{-4}$ \\
\hline
\end{tabular}

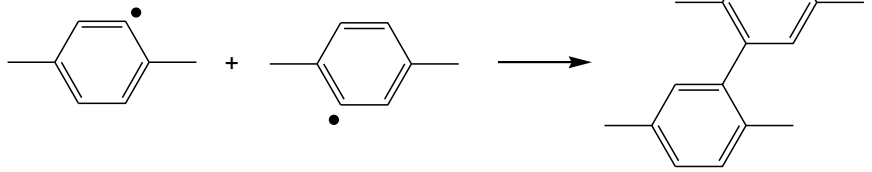

Fig. 20. Mécanisme de réticulation du PEEK par couplage intermoléculaire des radicaux phényle [3-6].

Fig 20. Crosslinking mechanism of PEEK by intermolecular coupling of phenyl radicals [3-6]. d'oxygène car, dans ces conditions d'exposition, tous les radicaux $\mathrm{P}^{\bullet}$ ont été quasi-instantanément transformés en radicaux $\mathrm{PO}_{2} \bullet$.

Une possibilité serait la terminaison par couplage intermoléculaire des radicaux péroxy. En effet, cette réaction implique, tout d'abord, la formation d'un pont tétraoxyde (POOOOP) très instable, qui se décompose quasi-instantanément à haute température (en particulier, au-dessus de $150^{\circ} \mathrm{C}$ ) pour donner deux radicaux $\mathrm{PO}^{\bullet}$ et de l'oxygène. Cependant, les radicaux $\mathrm{PO}^{\bullet}$ peuvent se réarranger pour finalement se coupler et ainsi, former un nœud de réticulation, comme illustré sur la figure 21.

Il existe d'autres mécanismes de réticulation qui peuvent se produire dans tout le domaine de pressions partielles d'oxygène. Le mécanisme de formation du benzoate de phényle a déjà été mentionné dans la section 3.1 (Fig. 7). Cependant, dans ce cas, on n'obtient pas un noeud de réticulation tétrafonctionnel (en «X»), mais trifonctionnel (en $« \mathrm{~T} »)$. On peut imaginer un autre mode de réarrangement du radical phénoxyle, impliquant la rupture d'une liaison cétone avant de se coupler avec le radical carboxyle pour donner, au final, l'anhydride benzö̈que (Fig. 22). Cependant, dans ce cas, on n'obtient pas un nœud de réticulation, mais on reforme une chaîne macromoléculaire.

Ces deux mécanismes jouent certainement un rôle majeur à l'échelle macromoléculaire, comme en témoigne la figure 23. En effet, il existe clairement une corrélation entre les accumulations du benzoate de phényle et de l'anhydride benzö̈que et l'augmentation de $\mathrm{T}_{\mathrm{g}}$. De plus, les vitesses d'accumulation de ces deux produits sont du même ordre de grandeur que la vitesse de réticulation.

\subsection{Conséquences de l'oxydation sur les propriétés élastiques}

Les profils de module élastique générés par l'oxydation dans l'épaisseur des plaquettes de $3 \mathrm{~mm}$ d'épaisseur de
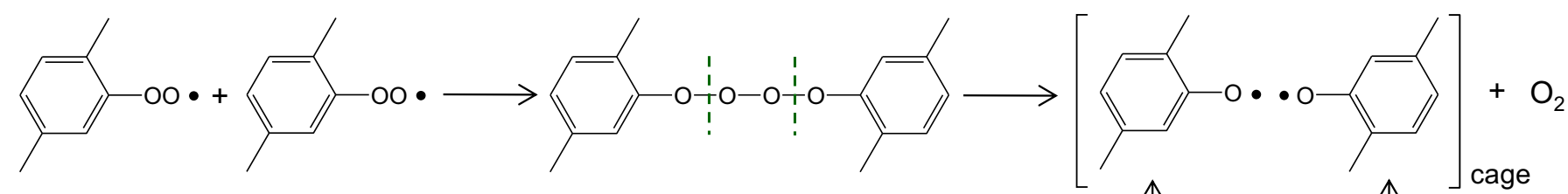<smiles>CC1=CC(=O)C(C)=C[CH+]1</smiles><smiles>C1CC1</smiles>

cage
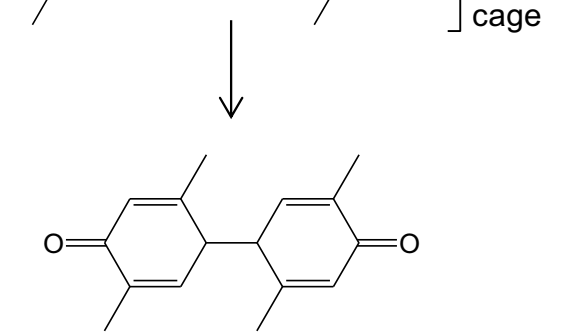

Fig. 21. Mécanisme de réticulation du PEEK par couplage intermoléculaire des radicaux péroxy.

Fig 21. Crosslinking mechanism of PEEK by intermolecular coupling of peroxy radicals. 

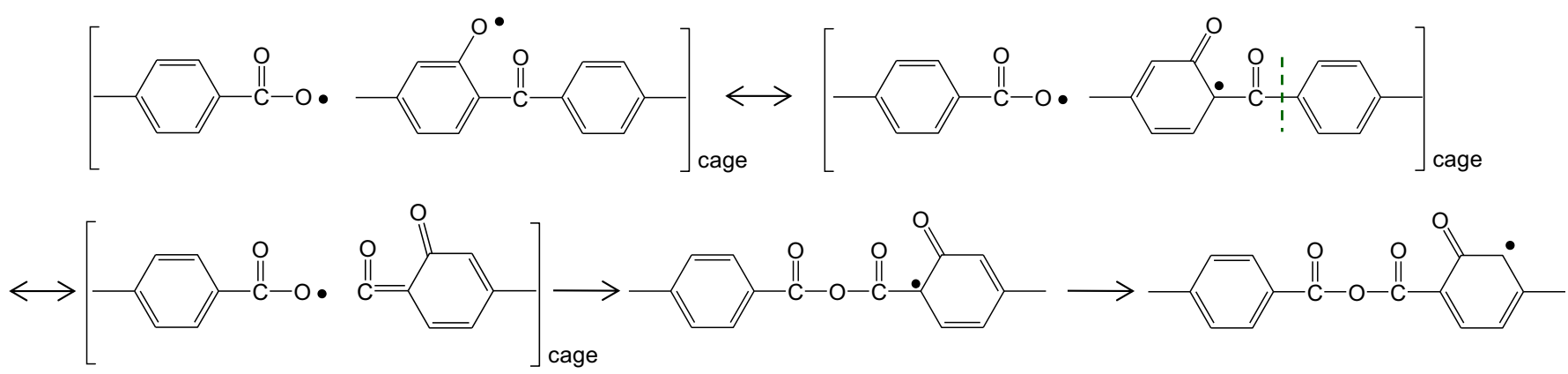

Fig. 22. Formation de l'anhydride benzoïque par couplage des radicaux carboxyle et phénoxyle.

Fig 22. Formation of benzoic anhydride by coupling of carboxyl and phenoxyl radicals.

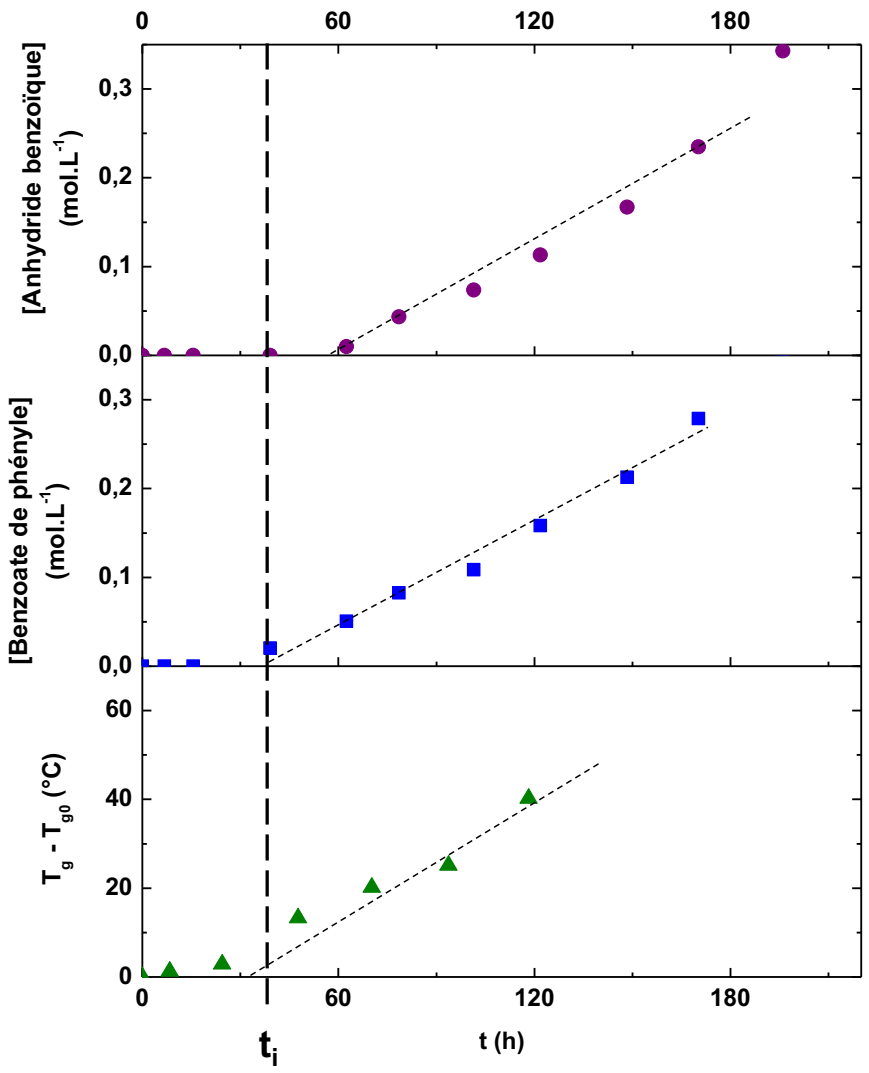

Fig. 23. Comparaison l'évolution des concentrations en anhydride benzoïque $(O)$ et en benzoate de phényle $(\square)$ avec l'évolution de la température transition vitreuse ( $\mathbf{\Delta}$ ) au cours du vieillissement thermique du PEEK sous 10 bars d'oxygène à $250^{\circ} \mathrm{C}$.

Fig 23. Comparison of the changes in the concentrations of benzoic anhydride (O) and phenyl benzoate $\square$ with the changes in the glass transition temperature ( $\mathbf{\Lambda}$ ) during the thermal ageing of PEEK under 10 bars of oxygen at $250^{\circ} \mathrm{C}$.

PEEK ont été déterminés par micro-indentation. À titre d'exemple, les profils obtenus avant et après $3166 \mathrm{~h}$ d'exposition dans l'air à $280^{\circ} \mathrm{C}$, et avant et après $645 \mathrm{~h}$ d'exposition dans l'air à $300^{\circ} \mathrm{C}$, sont rapportés sur la figure 24. On constate que le profil initial n'est pas tout à fait plat. En effet, le module élastique est légèrement plus faible en surface des échantillons en raison d'un taux de cristallinité plus faible (d'environ $30 \%$, contre $41 \%$ au coeur), résultant du refroidissement inhomogène des échantillons (gradient de température dans l'épaisseur) après leur mise en forme par injection. Néanmoins, une valeur moyenne de module élastique $\mathrm{E}_{\mathrm{Echant}}$ a été calculée pour le PEEK non vieilli: elle vaut $5,0 \pm 0,5 \mathrm{GPa}$. Cette valeur est légèrement supérieure à la valeur du module d'Young déterminée par traction uniaxiale sur des éprouvettes de type haltère: 4,2 $\pm 0,1 \mathrm{GPa}$. Pour cette raison, dans cette étude, un facteur correctif de 0,84 a été systématiquement appliqué au module d'indentation pour remonter à la valeur du module d'Young.

$\mathrm{Au}$ cours du vieillissement thermique, la microindentation met en évidence deux types distincts de comportement de part et d'autre de la température du pied du pic de fusion, c'est-à-dire de part et d'autre de $\mathrm{T}_{\text {f onset }} \approx$ $290^{\circ} \mathrm{C}$ (Fig. 25).

Lorsque $\mathrm{T}<290^{\circ} \mathrm{C}$, le module d'Young augmente lentement dans toute l'épaisseur de l'échantillon (Fig. 24a). Cette augmentation est principalement attribuée au phénomène de recuit [26-30], même si le phénomène de chimicristallisation ne peut pas être complètement exclus. En effet, l'augmentation de la mobilité moléculaire favorise la cristallisation des chaînes macromoléculaires dans la phase amorphe situées au voisinage de germes ou de petits cristaux quasiment indétectables par DSC. Quoi qu'il en soit, le recuit se manifeste par l'apparition d'un pic de fusion secondaire centré environ $20^{\circ} \mathrm{C}$ au-dessus de la température d'exposition sur les thermogrammes DSC. Ce pic se décale progressivement vers les hautes températures pour finalement s'intégrer au pic de fusion principal (Fig. 26a). Il en résulte une augmentation du taux global de cristallinité jusqu'à une valeur maximale de l'ordre de $50 \%$ (Fig. 26b). Des analyses de diffraction des rayons $\mathrm{X}$ en temps réel (c'est-à-dire à la température d'exposition) permettraient certainement de mieux comprendre l'origine et la croissance de ces nouveaux cristaux.

Lorsque $\mathrm{T}>290^{\circ} \mathrm{C}$, on observe une augmentation du module d'Young au coeur des échantillons, mais aussi une diminution du module d'Young dans la couche superficielle oxydée (Fig. 24b). Ici aussi, l'augmentation du module d'Young au cœur des échantillons est principalement attribuée au phénomène de recuit. En effet, à ces températures, les plus petits cristaux (c'est-à-dire les moins parfaits) fondent et ainsi, augmentent la fraction de phase amorphe susceptible de mieux cristalliser. On assiste 

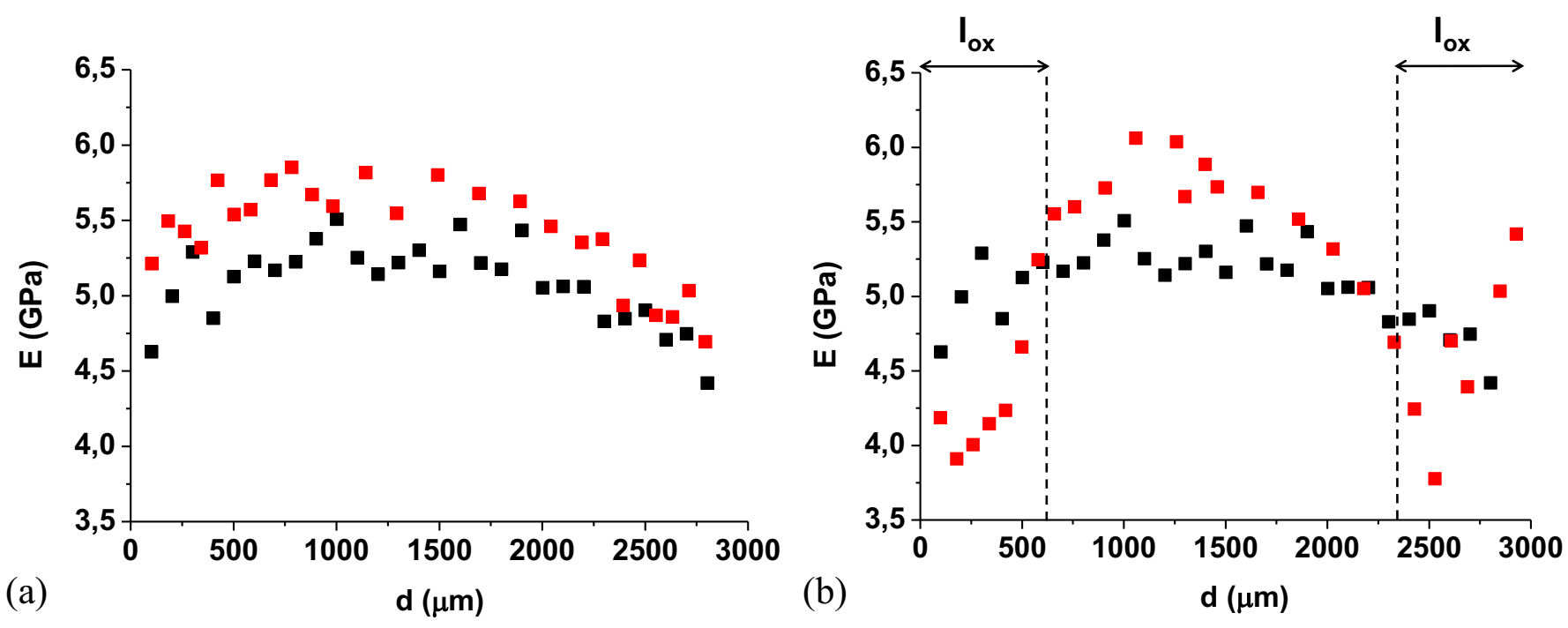

Fig. 24. Profils de module d'Young dans l'épaisseur d'une plaquette de PEEK avant (en noir) et après (rouge) $3166 \mathrm{~h}$ d'exposition dans l'air à $280^{\circ} \mathrm{C}$ (a) et $645 \mathrm{~h}$ d'exposition dans l'air à $300^{\circ} \mathrm{C}(\mathrm{b})$.

Fig 24. Profiles of the Young's modulus in the thickness of the PEEK plate before (in black) and after (red) 3166 h of exposure in air at $280^{\circ} \mathrm{C}(\mathrm{a})$ and $645 \mathrm{~h}$ of exposure in air at $300^{\circ} \mathrm{C}(\mathrm{b})$.

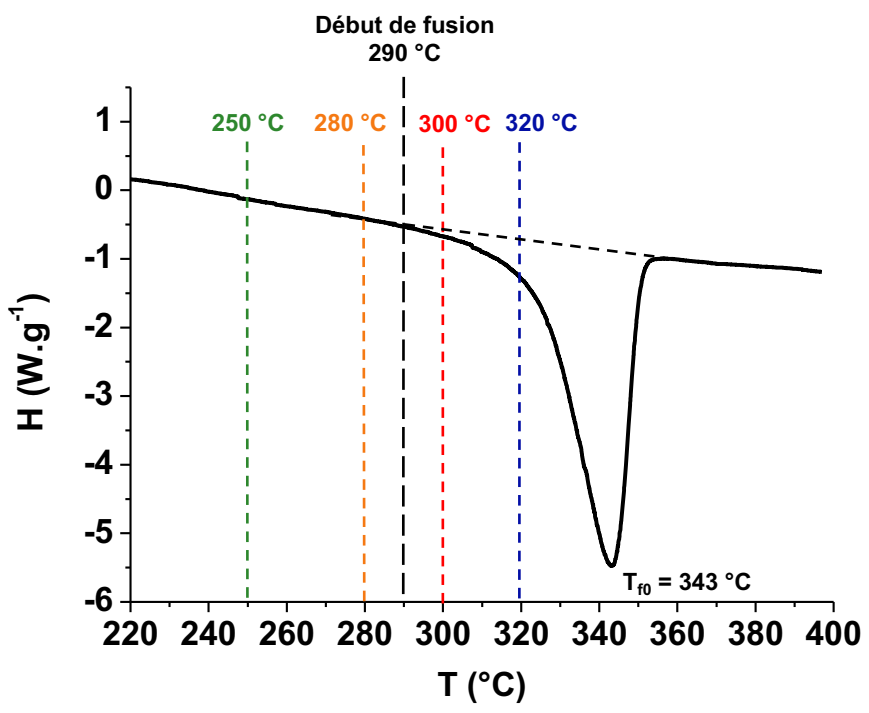

Fig. 25. Thermogramme DSC du PEEK avant vieillissement. Positionnement des températures de vieillissement thermique de chaque côté du pied de l'endotherme de fusion.

Fig 25. DSC thermogram of PEEK before ageing. Positioning of the thermal ageing temperatures on both sides of the onset of the melting endotherm.

alors à la disparition quasi-immédiate de la partie «basse température» du pic de fusion et, en contre-partie, à un soulèvement progressif de sa partie «haute température». Il en résulte une augmentation du taux de cristallinité jusqu'à une valeur maximale de l'ordre de $50 \%$. Soulignons que, en l'absence d'oxygène, les coupures de chaîne se produisant au niveau des liaisons cétone et éther (Fig. 11) détruisent progressivement le réseau d'enchevêtrements dans la phase amorphe et ainsi, libérent de courts segments de chaîne qui vont pouvoir facilement migrer jusqu'à la surface des cristaux restant pour les intégrer [31-32]. De ce point de vue, les coupures de chaîne favorisent le recuit de la matrice PEEK.

En revanche, en présence d'oxygène, rappelons que la réticulation prédomine largement sur les coupures de chaîne. Au terme d'une période d'induction d'environ $100 \mathrm{~h}$ dans l'air à $300^{\circ} \mathrm{C}$ (voir Fig. 17), la réticulation réduit considérablement la mobilité moléculaire dans la matrice PEEK et ainsi, s'oppose au phénomène recuit. Ainsi, le taux de cristallinité revient progressivement à sa valeur initiale (Fig. 27b).

Signalons que la diminution du module d'Young dans la couche oxydée se corrèle parfaitement avec les modifications de l'architecture macromoléculaire du PEEK, en particulier avec l'accumulation du benzoate de phényle déterminée par spectroscopie IRTF en mode ATR (Fig. 28).

Notons aussi que les épaisseurs de couche oxydée déterminées à l'aide de ces deux techniques (microindentation et spectroscopie IRTF) sont comparables. Elles sont du même ordre de grandeur que les épaisseurs de couche oxydée déterminées à l'aide d'une troisième technique: la microscopie optique. À titre d'exemple, la figure 29 présente des micrographies obtenues après quatre durées d'exposition dans l'air à $300^{\circ} \mathrm{C}$. On voit clairement que l'oxydation s'accompagne d'un changement de couleur (brunissement) en surface des échantillons.

Les épaisseurs moyennes de couche oxydée déterminées dans l'air à 280 et $300^{\circ} \mathrm{C}$ à l'aide de ces trois techniques sont récapitulées dans le tableau 6 .

Enfin, une corrélation a pu être établie entre le module d'Young et le taux de cristallinité. Les valeurs globales du module d'Young $\mathrm{E}_{\text {Echant }}$ des plaquettes de PEEK vieillies dans l'air à 280 et $300^{\circ} \mathrm{C}$ ont été calculées à l'aide de l'équation (6) à partir des profils de micro-indentation. Ces 

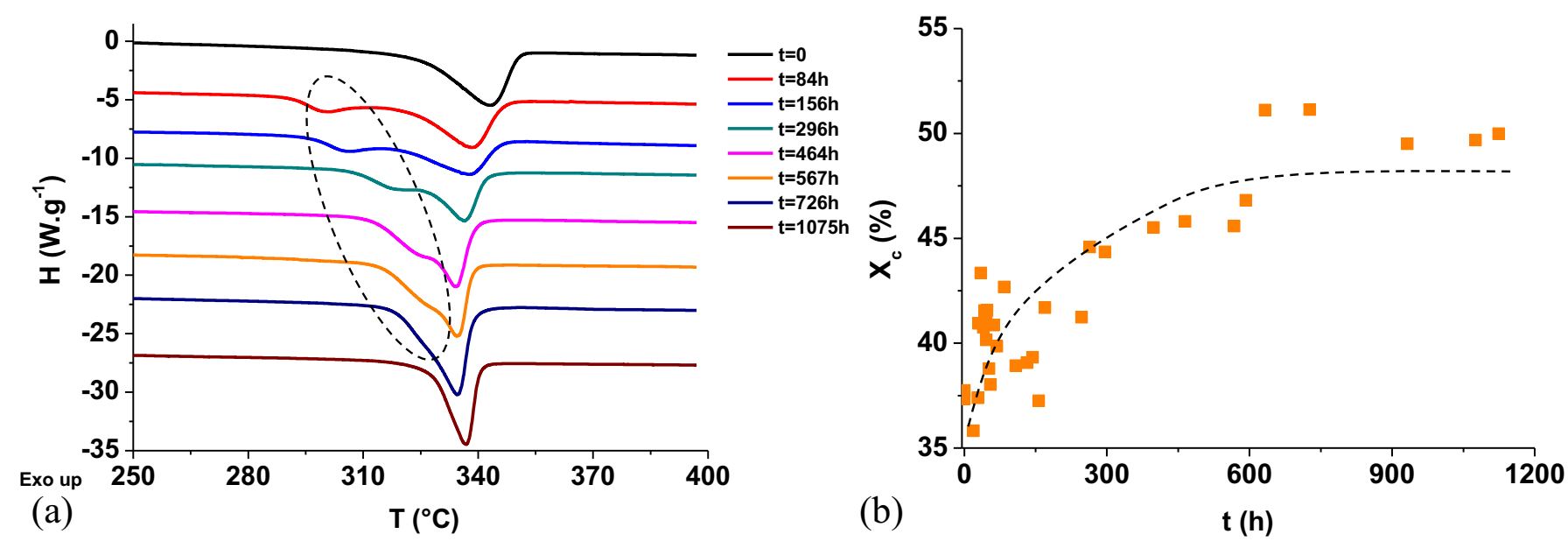

Fig. 26. Modification du thermogramme DSC (a) et évolution du taux de cristallinité (b) au cours du vieillissement thermique des films de PEEK dans l'air à $280^{\circ} \mathrm{C}$.

Fig 26. Modification of the DSC thermogram (a) and changes in the crystallinity ratio (b) during the thermal ageing of PEEK films in air at $280^{\circ} \mathrm{C}$.
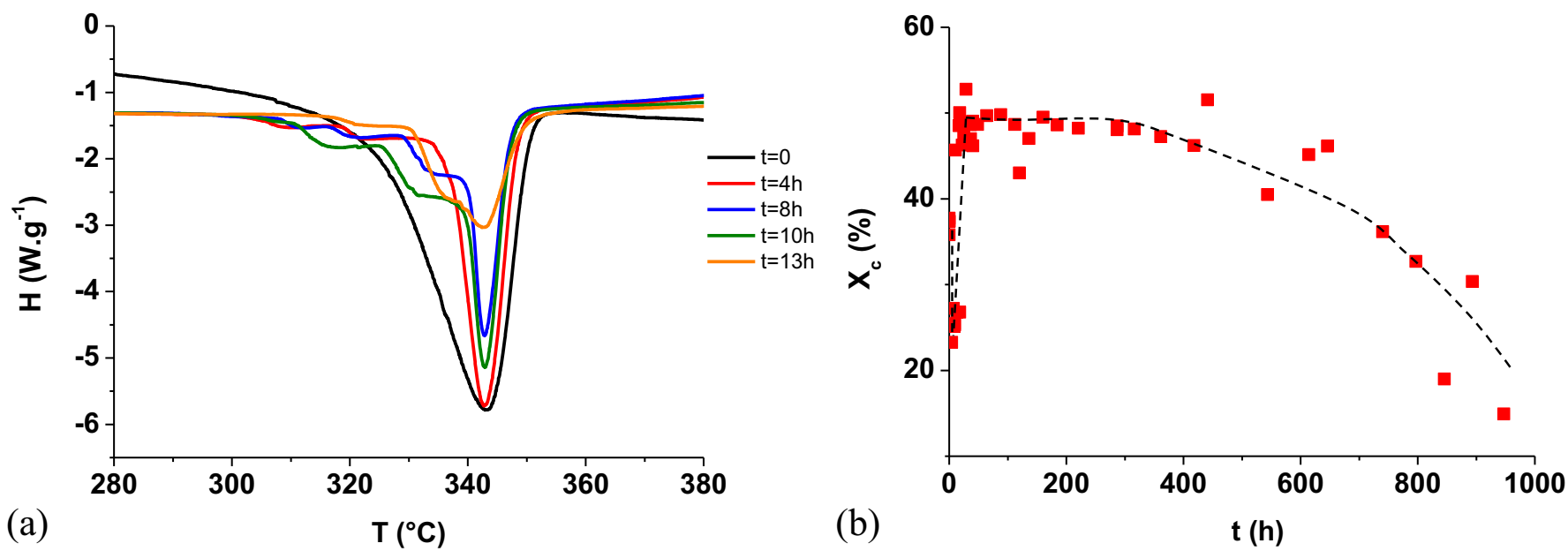

Fig. 27. Modification du thermogramme DSC (a) et évolution du taux de cristallinité (b) au cours du vieillissement thermique des films de PEEK dans l'air à $300^{\circ} \mathrm{C}$.

Fig 27. Modification of the DSC thermogram (a) and changes in the crystallinity ratio (b) during the thermal ageing of PEEK films in air at $300^{\circ} \mathrm{C}$.

valeurs ont été portées en fonction du taux de cristallinité $\mathrm{X}_{\mathrm{C}}$ sur la figure 30 , où elles sont comparées aux quelques données de traction uniaxiale disponibles dans la littérature $[33,34]$. On remarque que tous les points se placent autour d'une droite maîtresse unique d'équation:

$$
\frac{E_{\text {Echant }}}{E_{0}}=1+1,83.10^{-2} \times X c
$$

où $\mathrm{E}_{0}$ est le module d'Young de la phase amorphe du PEEK. Il vaut: $\mathrm{E}_{0}=3 \mathrm{GPa}$ en micro-indentation [17] et $\mathrm{E}_{0}=2,5 \mathrm{GPa}$ en traction uniaxiale [34]. [35]:

En fait, cette équation vérifie la relation de Tobolsky

$$
E_{\text {Echant }}=E_{0}+b \times \frac{\Delta H_{f 0} \times M}{V} \times X_{c}
$$

où $\mathrm{M}$ est la masse molaire et $\mathrm{V}$ le volume molaire du motif monomère, $\Delta \mathrm{H}_{\mathrm{f} 0}$ est l'enthalpie de fusion du cristal et b est une constante.

Rappelons que : $\mathrm{M}=288 \mathrm{~g} \cdot \mathrm{mol}^{-1}, \mathrm{~V}=206 \mathrm{~cm}^{3} \cdot \mathrm{mol}^{-1}$ et $\Delta \mathrm{H}_{\mathrm{f} 0}=130{\mathrm{~J} . \mathrm{g}^{-1}}^{-1}$ pour le PEEK [16]. En égalisant les équations (11) et (12), il vient finalement: $\mathrm{b}=25$. 


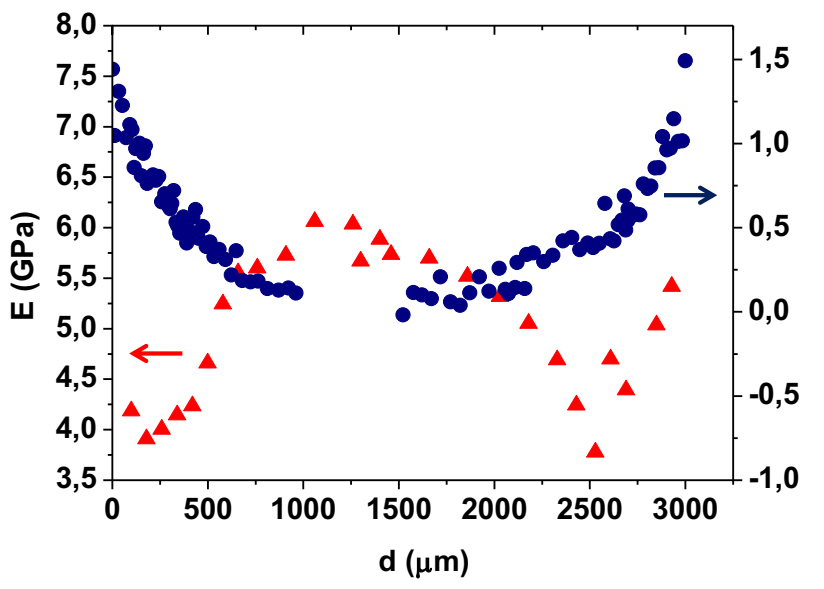

Fig. 28. Comparaison des profils de dégradation déterminés dans l'épaisseur d'une plaquette de PEEK par spectroscopie IRTF (O) et micro-indentation ( $(\mathbf{)})$ après $645 \mathrm{~h}$ d'exposition dans l'air à $300^{\circ} \mathrm{C}$.

Fig 28. Comparison of the degradation profiles determined in the thickness of a PEEK plate by FTIR spectrophotometry (O) and micro-indentation ( ) after $645 \mathrm{~h}$ of exposure in air at $300^{\circ} \mathrm{C}$.

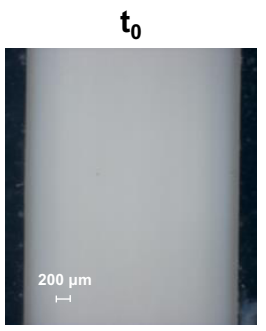

$381 \mathrm{~h}$

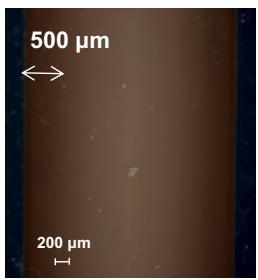

$65 \mathrm{~h}$

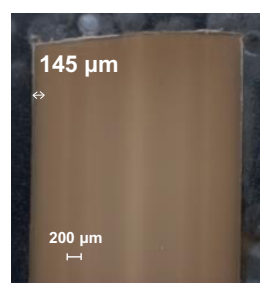

$501 \mathrm{~h}$

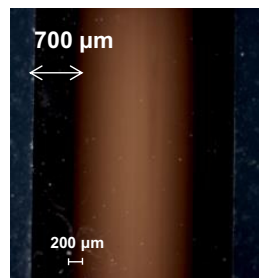

$193 \mathrm{~h}$

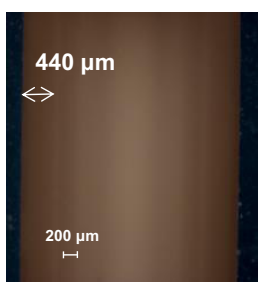

$645 \mathrm{~h}$

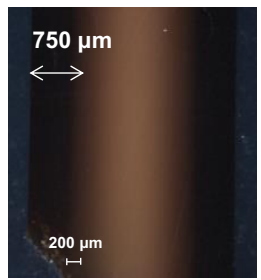

Fig. 29. Observation au microscope optique (avec un grossissement $\times 2,5$ ) de la couche superficielle oxydée du PEEK après différentes durées d'exposition dans l'air à $300{ }^{\circ} \mathrm{C}$.

Fig 29. Examination by optical microscopy (with a magnification of $\times 2,5)$ of the superficial oxidized layer of PEEK after different durations of exposure in air at $300^{\circ} \mathrm{C}$.

\section{Conclusion}

La dégradation thermique de la matrice PEEK a été étudiée à l'état caoutchoutique entre 180 et $320^{\circ} \mathrm{C}$ sous des pressions partielles d'oxygène comprises entre 0,21 et 50 bars, c'est-à-dire dans des domaines de température et de pression partielle d'oxygène presque complètement inexplorés. L'analyse à l'échelle moléculaire, par spectroscopie IRTF, a permis de d'identifier les principaux produits de dégradation s'accumulant dans la matrice PEEK
Tableau 6. Épaisseur moyenne de couche oxydée déterminée par micro-indentation, spectroscopie IRTF ou microscopie optique après différentes durées d'exposition du PEEK dans l'air à 280 et $300^{\circ} \mathrm{C}$.

Table 6. Average thickness of oxidized layer of PEEK determined by micro-indentation, FTIR spectrophotometry or optical microscopy after different durations of exposure in air at 280 and $300^{\circ} \mathrm{C}$.

\begin{tabular}{lrr}
\hline $\mathrm{T}\left({ }^{\circ} \mathrm{C}\right)$ & $\mathrm{t}(\mathrm{h})$ & $\mathrm{l}_{\mathrm{ox}}(\mu \mathrm{m})$ \\
\hline 280 & 381 & $105 \pm 15$ \\
& 1317 & $415 \pm 15$ \\
& 2492 & $620 \pm 30$ \\
300 & 3166 & $650 \pm 25$ \\
& 65 & $145 \pm 15$ \\
& 193 & $440 \pm 25$ \\
& 381 & $475 \pm 50$ \\
& 501 & $685 \pm 25$ \\
& 645 & $700 \pm 50$ \\
\hline
\end{tabular}

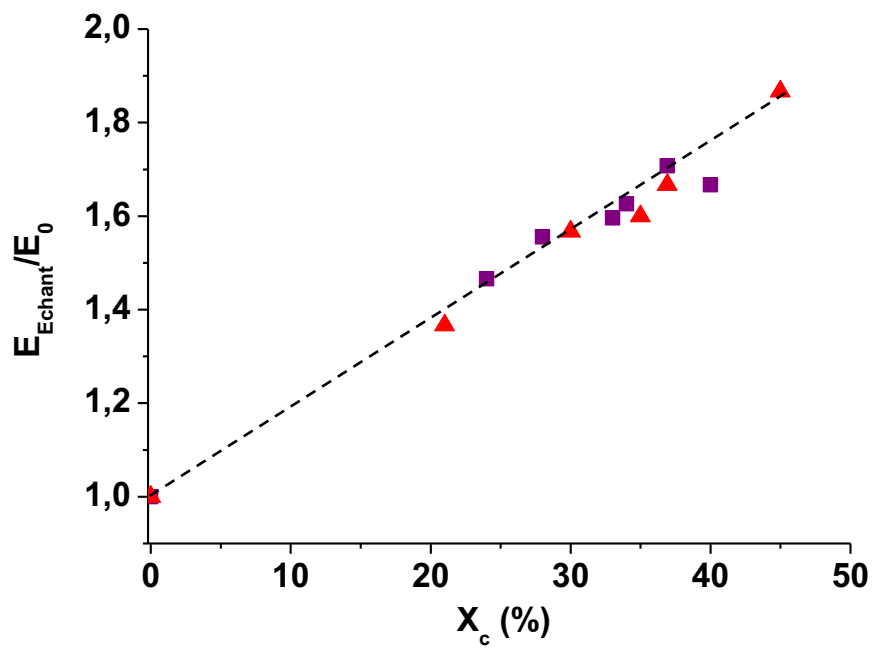

Fig. 30. Variation du module d'Young du PEEK (normalisé par le module de sa phase amorphe) avec le taux de cristallinité. Comparaison des valeurs déterminées par micro-indentation dans cetteétude (\) et traction uniaxiale dans la littérature [33,34]

Fig 30. Changes in the Young's modulus of PEEK (normalized by the Young's modulus of its amosphous phase) with crystallinity ratio. Comparison of the values determined by micro-indentation in the present study (М) and uniaxial tensile testing in the literature [33,34] ().

et d'élucider en partie les mécanismes de thermolyse et de thermo-oxydation. Ces produits sont l'anhydride benzoïque (à $\left.1780 \mathrm{~cm}^{-1}\right)$, le benzoate de phényle $\left(1740 \mathrm{~cm}^{-1}\right)$, la fluorénone $\left(1718 \mathrm{~cm}^{-1}\right)$, les phénols $\left(3650 \mathrm{~cm}^{-1}\right)$ et l'acide benzoïque $\left(3525 \mathrm{~cm}^{-1}\right)$. L'anhydride benzoïque et le benzoate de phényle sont les deux produits majoritaires sous forte pression partielle d'oxygène. Ils proviennent de la terminaison par couplage des radicaux carboxyle et phénoxyle. 
L'analyse aux échelles supérieures, par DSC puis par micro-indentation, a permis de montrer d'une part, que la réticulation prédomine largement sur les coupures de chaîne quelles que soient les conditions d'exposition. À l'état solide, un recuit s'opère dans toute l'épaisseur des échantillons, conduisant à une augmentation à la fois du taux de cristallinité et du module d'Young. En revanche, au-dessus du pied du pic de fusion, la réticulation réduit considérablement la mobilité moléculaire dans la couche oxydée où elle s'oppose au phénomène de recuit et ainsi, provoque la chûte du taux de cristallinité et du module d'Young. Dans tous les cas, les variations du module d'Young et du taux de cristallinité se corrèlent parfaitement et vérifient la relation de Tobolsky.

Dans une prochaine communication, un modèle cinétique sera dérivé du schéma mécanistique d'oxydation du PEEK pour être interfacé avec les relations structure/propriété identifiées dans cette étude (en particulier, avec les équations (9) et (12)). Cet outil théorique permettra de prédire non seulement l'état de dégradation du PEEK, mais aussi l'évolution de ses propriétés thermomécaniques au cours du vieillissement thermique. La validité de cet outil sera vérifiée à partir de l'ensemble des données expérimentales récoltées dans cette étude.

\section{References}

1. X. Colin, G. Teyssèdre, M. Fois, Ageing and degradation of multiphase polymer systems, in: A. Boudenne, L. Ibos, Y. Candau, S. Thomas (Eds.), Handbook of Multiphase Polymer Systems V 2/2, John Wiley \& Sons Ltd, Chichester, 2011, pp. 797-841.

2. K.C. Cole, I.G. Casella, Fourier transform infrared spectroscopic study of thermal degradation in films of poly(etheretherketone), Thermochim. Acta. 211, 209 (1992)

3. M. Day, D. Sally, D.M. Wiles, Thermal degradation of poly (aryl-ether-ether-ketone): experimental evaluation of crosslinking reactions, J. Appl. Polym. Sci. 40(9-10), 1615 (1990)

4. J.N. Hay, D.J. Kemmish, Thermal decomposition of poly (aryl ether ketones), Polymer 28(12), 2047 (1987)

5. A. Jonas, R. Legras, Thermal stability and crystallization of poly(aryl ether ether ketone), Polymer 32(15), 2691 (1991)

6. P. Patel, T.R. Hull, R.W. McCabe, D. Flath, J. Grasmeder, M. Percy, Mechanism of thermal decomposition of poly(ether ether ketone) (PEEK) from a review of decomposition studies, Polym. Degrad. Stab. 95(5), 709 (2010)

7. C. Nicodeau, Modélisation du soudage en continu de composites à matrice thermoplastique, Thèse de doctorat, ENSAM, Paris, 2005

8. M. Day, T. Suprunchuk, J.D. Cooney, D.M. Wiles, Thermal degradation of poly(aryl-ether-ether-ketone) (PEEK): a differential scanning calorimetry study, J. Appl. Polym. Sci. 36(5), 1097 (1988)

9. K.L. White, L. Jin, N. Ferrer, M. Wong, T. Bremner, H.J. Sue, Rheological and thermal behaviors of commercial poly (aryletherketone)s, Polym. Eng. Sci. 53(3), 651 (2013)

10. E. Richaud, P. Ferreira, L. Audouin, X. Colin, J. Verdu, C. Monchy-Leroy, Radiochemical ageing of poly(ether ether ketone), Eur. Polym. J. 46(4), 731 (2010)
11. S. Giancaterina, A. Rossi, A. Rivaton, J.L. Gardette, Photochemical evolution of poly(ether ether ketone), Polym. Degrad. Stab. 68(1), 133 (2000)

12. S. Commereuc, D. Vaillant, J.L. Philippart, J. Lacoste, J. Lemaire, D.J. Carlsson, Photo and thermal decomposition of iPP hydroperoxides, Polym. Degrad. Stab. 57, 175 (1997)

13. D.J. Carlsson, R. Brousseau, D.M. Wiles, Reactions of sulfur dioxide with oxidized polyolefins, Polym. Degrad. Stab. 15, 67 (1986)

14. D.J. Carlsson, D.M. Wiles, The photodegradation of polypropylene films. II. photolysis of ketonic oxidation products, Macromolecules 2(6), 587 (1969)

15. F. Gugumus, Formation of ester functional groups in oxidizing polymers, Polym. Degrad. Stab. 65(1), 5 (1999)

16. D.J. Blundell, B.N. Osborn, The morphology of poly(arylether-ether-ketone), Polymer 24(8), 953 (1983)

17. T. Iqbal, B.J. Briscoe, P.F. Luckham, Surface plasticization of poly(ether ether ketone), Eur. Polym. J. 47(12), 2244 (2011)

18. W.C. Oliver, G.M. Pharr, An improved technique for determining hardness and elastic modulus using load and displacement sensing indentation experiments, J. Mater. Res. 7(06), 1564 (1992)

19. S. Collin, P.-O. Bussière, S. Thérias, J.-M. Lambert, J. Perdereau, J.-L. Gardette, Physicochemical and mechanical impacts of photo-ageing on bisphenol a polycarbonate, Polym. Degrad. Stab. 97(11), 2284 (2012)

20. S.J. Blanksby, G.B. Ellison, Bond dissociation energies of organic molecules, Acc. Chem. Res. 36(4), 255 (2003)

21. L.K. Nait-Ali, X. Colin, A. Bergeret, Kinetic analysis and modelling of PET macromolecular changes during its mechanical recycling by extrusion, Polym. Degrad. Stab. 96(2), 236 (2011)

22. J. Pospísil, Chemical and photochemical behaviour of phenolic antioxidants in polymer stabilization: a state of the art report, part II, Polym. Degrad. Stab. 39(1), 103 (1993)

23. X. Colin, L. Audouin, J. Verdu, Determination of thermal oxidation rate constants by an inverse method. Application to polyethylene, Polym. Degrad. Stab. 86(2), 309 (2004)

24. T.G. Fox, P.J. Flory, Second-order transition temperatures and related properties of polystyrene. I. Influence of molecular weight, J. Appl. Phys. 21(6), 581 (1950)

25. D.W.V. Krevelen, K.T. Nijenhuis, Properties of Polymers, vol. Fourth completely revised edition, Elsevier, Amsterdam, 2009

26. D.J. Blundell, On the interpretation of multiple melting peaks in poly(ether ether ketone), Polymer 28(13), 2248 (1987)

27. G.M.K. Ostberg, J.C. Seferis, Annealing effects on the crystallinity of polyetheretherketone (PEEK) and its carbon fiber composite, J. Appl. Polym. Sci. 33(1), 29 (1987)

28. D.C. Bassett, R.H. Olley, I.A.M. Al Raheil, On crystallization phenomena in PEEK, Polymer 29(10), 1745 (1988)

29. P. Cebe, Annealing study of poly(etheretherketone), J. Mater. Sci. 23(10), 3721 (1988)

30. M. Buggy, A. Carew, The effect of thermal ageing on carbon fibre-reinforced polyetheretherketone (PEEK) - Part II Morphological changes, J. Mater. Sci. 29(8), 2255 (1994)

31. M.S. Rabello, J.R. White, Crystallization and melting behaviour of photodegraded polypropylene-I. Chemi-crystallization, Polymer 38(26), 6379 (1997)

32. B. Fayolle, E. Richaud, X. Colin, J. Verdu, Review: degradation-induced embrittlement in semi-crystalline 
polymers having their amorphous phase in rubbery state, J. Mater. Sci. 43(22), 6999 (2008)

33. T. Nishino, K. Tada, K. Nakamae, Elastic modulus of crystalline regions of poly(ether ether ketone), poly(ether ketone) and poly(p-phenylene sulphide), Polymer 33(4), 736 (1992)
34. N.D. Albérola, P. Mélé, C. Bas, Tensile mechanical properties of PEEK films over a wide range of strain rates. II, J. Appl. Polym. Sci. 64(6), 1053 (1997)

35. A.V. Tobolsky, Properties and structure of polymers, Wiley, New York, 1960

Citation de l'article : Emilie Courvoisier, Yoann Bicaba, Xavier Colin, Analyse de la dégradation thermique du Poly(éther éther cétone), Matériaux \& Techniques 105, 403 (2017) 